\title{
Efectividad de los Equipos de Trabajo, una Revisión de la Última Década de Investigación (1999-2009)
}

\section{Work Team Effectiveness, a Review of Research over the last Decade (1999-2009)}

\author{
Ramón Rico \\ Universidad Autónoma de Madrid
}

\author{
Carlos María Alcover de la Hera \\ Universidad Rey Juan Carlos de Madrid
}

\author{
Carmen Tabernero \\ Universidad de Córdoba
}

\begin{abstract}
Resumen. Los equipos no siempre aportan la diversidad en conocimientos, actitudes, habilidades y experiencias que permite ofrecer respuestas innovadoras a los retos planteados alcanzando el rendimiento que se espera de ellos. Este artículo sintetiza e integra los principales resultados de la investigación sobre equipos de trabajo realizada en la década comprendida entre los años 1999 y 2009. Con este objetivo el artículo expone de forma breve un marco explicativo de la efectividad de los equipos de trabajo a través de un análisis diferenciado de insumos, mediadores y resultados. Para ello, se utiliza una aproximación basada en el método DAFO, según el cual se identifican las fortalezas, debilidades, amenazas y oportunidades que presenta la investigación sobre equipos de trabajo de cara a la década venidera. Finalmente se discuten de manera integrada los principales retos que este ámbito de investigación tiene ante sí para convertir las amenazas en oportunidades.

Palabras clave: equipos de trabajo, efectividad, método DAFO, insumos, mediadores.

Abstract. Teams do not always provide the diversity of knowledge, attitudes, skills and experience required to generate an innovative response to challenges or perform according to expectations. This paper synthesizes the key results of research into work teams carried out over the last decade from 1999 through 2009.To this end, we set out a brief explanatory framework for the effectiveness of work teams based on a differentiated analysis of inputs, mediators and outcomes. Our approach uses the SWOT technique, which identifies strengths, weaknesses, opportunities and threats with a view to teamwork research in the next decade. Finally, we integrate and discuss the key challenges facing the field if it is to change threats into opportunities.

Keywords: work teams, effectiveness, SWOT technique, inputs, mediators.
\end{abstract}

Los equipos se han convertido durante las últimas cuatro décadas en un elemento central en el funcionamiento de las organizaciones. Este hecho ha sido facilitado, al menos en parte, por distintos trabajos que informan de las relaciones positivas entre el trabajo basado en equipos y la calidad de los productos y servicios ofrecidos por una organización (Gibson, Porath, Benson y Lawler, 2007). Son, no obstante, las presiones derivadas de la necesidad de configurar modelos de negocio cambiantes en ambientes dinámicos, inciertos y complejos y la necesidad de innovación, las que exigen adaptar la estructura del trabajo tradicionalmente configurada en torno a los individuos y adoptar diseños organizacionales orientados al cambio y basados en equipos (Lawler y Worley, 2006; West y Markiewicz, 2004). Y esta tendencia se ha comprobado en todo tipo de contextos laborales, tanto privados

La correspondencia sobre este artículo debe enviarse al primer autor a la dirección: c/ Iván Pavlov, 6, Facultad de Psicología, Universidad Autónoma de Madrid, 28049, Madrid. e-mail: ramon.rico@uam.es como públicos, incluido el ámbito militar (Kozlowski e Ilgen, 2006).

Los equipos aportan la diversidad en conocimientos, actitudes, habilidades y experiencias cuya integración permite ofrecer respuestas rápidas, flexibles e innovadoras a los problemas y retos planteados, promoviendo el rendimiento y mejorando la satisfacción de sus integrantes. Esto es el resultado de lo que se ha denominado la sabiduría de los colectivos: una capacidad incrementada para lograr diversos tipos de rendimiento posibilitada por las interacciones de los miembros (Salas, Rosen, Burke y Goodwin, 2009). De este modo, el éxito de las organizaciones y la producción global de conocimiento depende en gran medida de la eficacia de los equipos (Wuchty, Jones y Uzzi, 2007).

Sin embargo los equipos no siempre actúan así, y en ocasiones no logran alcanzar el elevado rendimiento que se espera de ellos (Sims, Salas y Burke, 2005). De hecho, si atendemos a nuestra experiencia cotidiana, es frecuente que los equipos, lejos de convertirse en mecanismos de aprovechamiento eficaz y satisfactorio 
de esfuerzos colectivos, se transformen en agujeros negros que inmisericordemente consumen las energías físicas, mentales y emocionales de sus integrantes. Esto suele ocasionar un derroche de esfuerzos para el logro de sus metas o, sencillamente, que no se consigan en absoluto. Por tanto, el desafío para la investigación y la intervención radica en la eficaz integración de las aportaciones de personas cualificadas y expertas que aporten un valor añadido a la organización. Sin olvidar el importante papel que desempeña el diseño del contexto organizacional en el que todo equipo se inserta, de manera que facilite la creación de estructuras y líneas de apoyo, comunicación, consulta, retroalimentación y recompensas que complementen el funcionamiento interno del equipo (Aritzeta y Alcover, 2006; Hackman, 1998).

Al mismo tiempo que las organizaciones, debido a las razones prácticas señaladas, han incrementado sus experiencias en la utilización de equipos, también se ha detectado un significativo aumento en la investigación orientada al desarrollo de métodos y teorías para conocer la eficacia de los equipos de trabajo (Goodwin, Burke, Wildman y Salas, 2009). Esto ha traído consigo la proliferación de modelos y constructos que tratan de describir, comprender y explicar el funcionamiento de los equipos de trabajo, si bien la dispersión teórica y metodológica ha dificultado la integración y consolidación de los resultados obtenidos (Weingart y Cronon, 2009). Por ejemplo, siguiendo la revisión realizada hace ya algunos años por Cannon-Bowers, Tannenbaum, Salas y Volpe (1995), Salas, Stagl, Burke y Goodwin (2007) han identificado recientemente138 propuestas procedentes de diferentes disciplinas que tratan de definir los procesos y habilidades grupales implicados en el rendimiento y la eficacia de los equipos. Y aunque también ha habido intentos de reducir esta enorme dispersión -como la imaginativa propuesta de Salas, Sims y Burke (2005), quienes sugieren la posibilidad de identificar un constructo similar al big five relacionado con el trabajo en equipo-, parece que nos encontramos lejos aún de lograr una deseable integración (Salas y Wildman, 2009; Weingart y Cronon, 2009).

Este artículo tiene como principal objetivo sintetizar e integrar los principales resultados de la investigación sobre equipos de trabajo realizada en la década comprendida entre los años 1999 y 2009.Para ello, utilizando una aproximación basada en el método DAFO, identificaremos las fortalezas, debilidades, amenazas y oportunidades que presenta el ámbito de investigación de cara a la década venidera. Con este objetivo expondremos de forma breve un marco explicativo de la efectividad de los equipos de trabajo que nos permitirá un análisis diferenciado de insumos, mediadores y resultados. Finalmente discutiremos de manera integrada los principales retos que este ámbito de investigación tiene ante sí para convertir las amenazas en oportunidades.

\section{Marco explicativo de la eficacia de los equipos de trabajo}

Podemos definir los equipos de trabajo como colectivos que existen para realizar tareas organizacionalmente relevantes, que mantienen un cierto grado de interdependencia tanto en términos de objetivos como de tarea, gestionan y mantienen sus límites y están inmersos en un contexto organizacional que limita su actividad e influye sobre sus intercambios con otros equipos dentro de la organización (Kozlowski y Bell, 2003).

Son numerosas y recientes las revisiones sobre la investigación relativa a la efectividad de los equipos en los últimos diez años (Gil, Alcover y Peiró, 2005; Goodwin et al., 2009; Ilgen, Hollenbeck, Johnson y Jundt, 2005; Kozlowski y Bell, 2003; Kozlowski e Ilgen, 2006; Mathieu, Maynard, Rapp y Gilson, 2008; Nielsen, Sundstrom y Halfhill, 2005; Salas, Stagl y Burke, 2004; Sundstrom, McIntyre, Halfhill y Richards, 2000) y, aunque con matices, en su totalidad han sido realizadas bajo el esquema que marca el modelo Input-Procesos-Output (IPO) (McGrath, 1964). Este modelo identifica la composición, la estructura y los procesos de los equipos como los antecedentes clave de la eficacia del equipo. Asimismo, el modelo señala que los factores organizacionales y situacionales influyen en la estructura del equipo como un todo, afectando a las demás variables (input, proceso, output).

El modelo IPO ha recibido importantes críticas centradas, primero, en su incapacidad para incorporar los aspectos temporales y recursivos que el desarrollo y la retroalimentación imponen sobre los equipos, con el consiguiente olvido de los procesos adaptativos e incrementales de aprendizaje de equipo que influyen necesariamente sobre su eficacia (véase a este respecto, por ejemplo, la propuesta de Kozlowski, Gully, Nason y Smith, 1999); y segundo, en su tratamiento unitario, simplificado y opaco de los procesos de equipo. Estas críticas han motivado que en los últimos años surjan modelos alternativos que intentan reflejar mejor el funcionamiento de los equipos como sistemas adaptativos complejos operando en contextos más amplios (ej. una organización). Así, el modelo CORE (véase McGrath, Arrow y Berdahl, 2000) explica el desarrollo de los equipos a lo largo del tiempo, identificando sus procesos básicos (construcción, operaciones, reconstrucción y relaciones externas) y considerando las relaciones con el contexto del equipo, o la aproximación THEDA (Team Holistic Ecology Dynamic Activity) (Cooke, Gorman y Winner, 2007) que busca la aplicabilidad a equipos heterogéneos y la diferenciación de enfoques sobre proceso frente a enfoques sobre conocimientos. Por su parte, el modelo IMOI (InputMediador-Output-Input; Ilgen et al., 2005), tal como recoge la Figura 1, señala el carácter cíclico de los procesos de retroalimentación, de forma que los outputs 
del equipo en un momento dado representan nuevos inputs para actuaciones posteriores. Kozlowski e Ilgen (2006) han integrado estas ideas en un modelo que considera a los equipos como sistemas multinivel (nivel individual, de equipo y organizacional), orientados a los procesos relevantes para la tarea y que evolucionan con el tiempo, de forma que tanto los procesos como la eficacia de los equipos constituyen fenómenos emergentes (patrones resultantes de la interacción regular y repetida entre los miembros).

El modelo reflejado en la Figura 1 nos ofrece el esquema con el que vamos a guiar nuestra revisión y análisis de la literatura sobre efectividad de equipos en la última década. Avanzaremos de izquierda a derecha y analizaremos cada una de las grandes categorías (insumos, mediadores y resultados) utilizando el marco del método DAFO. No es nuestra intención trasponer una herramienta de análisis estratégico a este ámbito particular de investigación, tan sólo utilizaremos el análisis DAFO como pretexto creativo para emular un análisis de las fortalezas, debilidades, amenazas y oportunidades que nos guíen estratégicamente en el desarrollo futuro de investigación en el ámbito. mos, pueden constituir elementos facilitadores o inhibidores de la efectividad de los equipos. En la revisión que planteamos vamos a transitar del nivel más externo al más interno.

El contexto de la organización tiene un papel crítico en la eficacia de los equipos (Hackman, 2002). En el modelo IMOI, el contexto organizacional se representa mediante tres tipos de apoyo que ofrece a los equipos: 1) los sistemas de dirección de RR.HH. (formación, evaluación del desempeño, compensación...); 2) el diseño de la organización que permite interrelacionar los equipos de trabajo y a estos con el conjunto de la organización, articula los flujos de transmisión de información y coordinación y permite apoyos, que facilitan recursos y eliminan obstáculos y 3) el clima organizacional de apoyo a los equipos y la cultura organizacional (no deberíamos descartar la cultura nacional).

Los resultados de investigaciones recientes ponen de manifiesto el grado en que el alineamiento de las políticas de RR.HH. con relación a las particularidades de los equipos (ej. usar complementariamente recompensas individuales y grupales para reforzar el rendi-

Figura 1. Modelo IMOI (Adaptado de Mathieu et al., 2008)

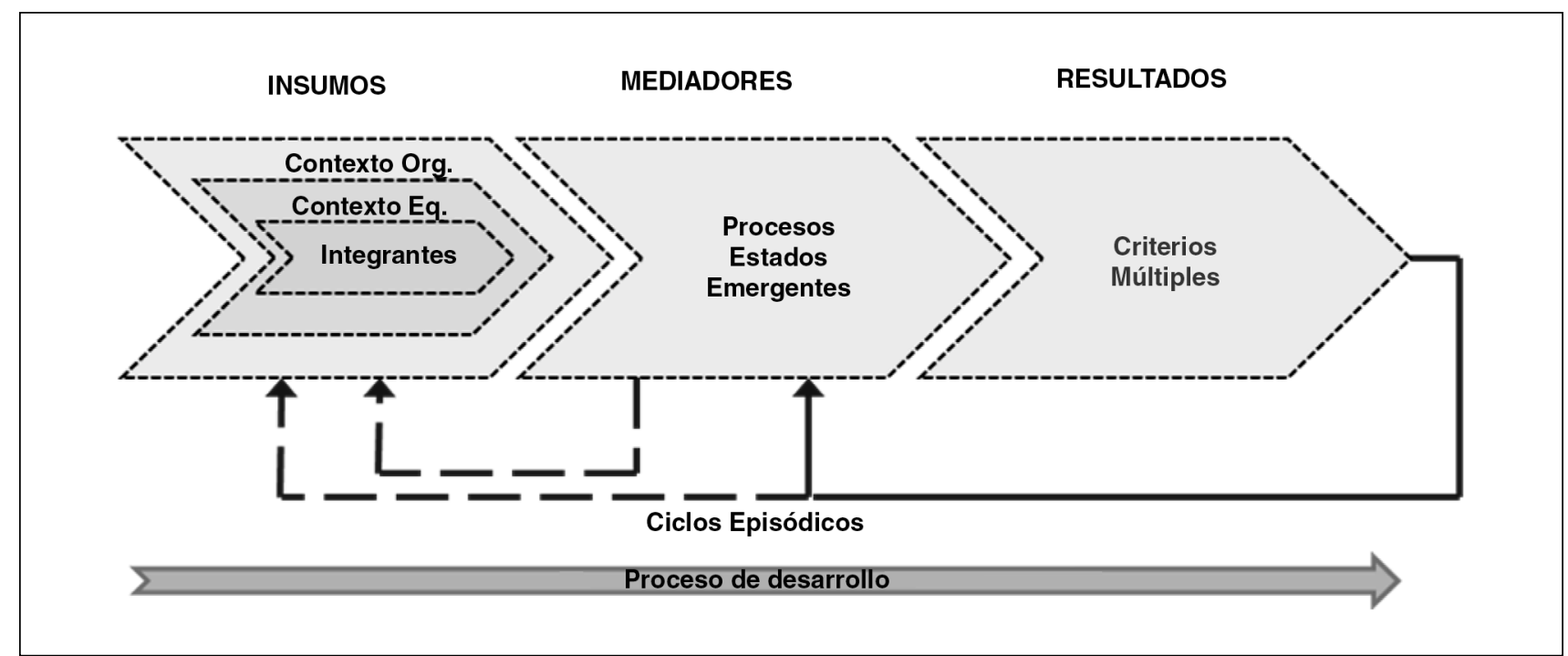

\section{Insumos: recursos, tarea y composición del equipo}

Los insumos representan el conjunto de recursos del equipo, tanto externos (ej. las recompensas de la organización, la cultura organizacional), como internos (la composición del equipo, fundamentalmente los conocimientos y habilidades de sus miembros, así como la estructura del grupo y el diseño de la tarea). Así mismo, estos recursos pueden considerarse a distintos niveles (recursos de los miembros, del grupo y de la organización). Es importante considerar que los insu- miento de los equipos) produce un efecto indirecto sobre su efectividad (Hyatt y Ruddy, 1997), al tiempo que se relaciona directamente con la capacidad de autogestión que es capaz de asumir el equipo (Kirkman y Rosen, 1999). Respecto al impacto de las políticas formativas, un reciente meta-análisis confirmó una correlación de .29 para la influencia global que ejerce la formación en los equipos en la mejora tanto del rendimiento objetivo de los equipos, como de su evaluación por parte de los supervisores (Salas, Nichols y Driskell, 2007).Así mismo, existe una larga tradición 
en identificar el papel que juega el diseño organizacional sobre los equipos de trabajo a la hora de generar sistemas de alta implicación y de coordinación entre equipos. Con relación a los sistemas de coordinación entre equipos (multi-teamsystems) la literatura reciente muestra que la efectiva coordinación entre equipos predice beneficios sobre el rendimiento de los equipos y sobre sus procesos internos, especialmente en entornos de alta interdependencia (Marks, Mathieu, Alonso, DeChurch, y Panzer 2005; Mathieu et al., 2006; van der Vegt y van der Vliert, 2005). Respecto a los sistemas de alta implicación, éstos son señalados como especialmente relevantes para los diseños orientados al cambio tan necesarios en la actual coyuntura socioeconómica (Lawler y Worley, 2006). Sistemas de alta implicación que han mostrado ser buenos predictores de la productividad en equipos (Spreitzer, Cohen y Ledford, 1999) e influir especialmente en el clima organizacional. A este respecto, son varios los estudios que apuntan cómo el clima de apertura tiene efectos multinivel, facilitando los procesos grupales y la participación en los procesos de toma de decisiones (Mathieu, Maynard, Taylor, Gilson y Rudy, 2007; Tesluk, et al., 1999). Por su parte, otros estudios se han centrado en analizar el papel desempeñado por la percepción de apoyo organizacional por parte de los miembros, encontrándose que tiene una influencia positiva sobre la potencia de los equipos, si bien dicha relación se encuentra mediada por los procesos grupales (Kennedy, Loughry, Klammer y Beyerlein, 2009). Finalmente, la investigación sobre el papel que el contexto cultural más amplio genera sobre la organización está emergiendo y los pocos estudios que aparecen, reflejan cómo las diferencias culturales nacionales afectan a la cultura de calidad en la organización y la calidad de servicio ofrecida por sus equipos (Gibson, 2003)

Diseño de la tarea y el contexto de trabajo de los equipos: La autonomía es una de las características que más atención ha recibido en los últimos años. La autonomía se refiere al grado en que un equipo tiene capacidad para tomar decisiones sobre diferentes aspectos de su trabajo (métodos, horarios, roles, etc.). Un nivel bajo de autonomía indica que el equipo tiene una tarea muy estructurada y definida por la organización, lo que minimiza la necesidad de tomar decisiones colectivas o gestionar los procesos internos. Por el contrario, un nivel alto de autonomía implica que los miembros del equipo deben tomar numerosas decisiones colectivamente sobre su trabajo. Los estudios concluyen que la autonomía es una característica básica del diseño de la tarea de los equipos que modula los efectos tanto de otras variables antecedentes (p. ej. diversidad del equipo) como de los procesos (ej. manejo del conflicto) sobre la eficacia grupal (Kozlowski y Bell, 2003). En un reciente meta-análisis sobre las relaciones entre el diseño del equipo y su rendimiento (Stewart, 2006) se encontró que el incremento de auto- nomía junto con la coordinación dentro del equipo se correspondía con el mayor rendimiento, si bien el tamaño del efecto variaba dependiendo del tipo de tarea.

También la interdependencia, como característica de la tarea del equipo, ha acaparado la atención de numerosos investigadores (Stewart y Barrick, 2000). Se han identificado distintos tipos de interdependencia, pero las principales tienen que ver con tarea y objetivos. La interdependencia de tarea es el grado en que los miembros de un equipo dependen unos de otros e interactúan entre sí para lograr sus objetivos. La interdependencia de objetivos es el grado en que los miembros de un equipo comparten objetivos en su actividad. Los estudios centrados sobre la interdependencia de tarea han mostrado su efecto modulador (generalmente en términos positivos) sobre muchos de los efectos que distintos procesos de equipo ejercen sobre los resultados organizacionales, como por ejemplo las conductas de ayuda (Bachrach, Powell, Collins y Richey, 2005), la confianza (Rico, Alcover, Sánchez-Manzanares y Gil, 2009), la comunicación, el conflicto o la flexibilidad (Stewart y Barrick, 2000). Así mismo, estos efectos moduladores se extienden a los efectos directos que otras variables de tarea tienen sobre el rendimiento, como son la virtualidad (Rico y Cohen, 2005) o la propia autonomía. A este respecto Langfred (2005) muestra cómo los efectos positivos de la autonomía sobre el rendimiento de equipo sólo se producen bajo condiciones de alta interdependencia. Por su parte, la interdependencia de objetivos ha mostrado relaciones positivas con un mayor aprendizaje, cantidad de información compartida y efectividad del equipo (De Dreu, 2007). Adicionalmente, la consideración conjunta de interdependencias de tarea y objetivos ha dado lugar a la denominada interdependencia compleja. En ella se enmarca la hipótesis de la congruencia que da cuenta de los efectos interactivos de ambas variables sobre aspectos tan diversos como las respuestas afectivas o los comportamientos de innovación (van der Vegt, Emans y van der Vliert, 2005).

La virtualidad ha sido objeto de un número creciente de estudios en la primera parte de esta década. De hecho, hemos de reconocer que todos nosotros trabajamos con un determinado grado de virtualidad. La virtualidad se define a partir de tres dimensiones: dependencia de los miembros respecto de las tecnologías de la información y comunicación para coordinase y ejecutar los procesos del equipo, tipos de información que aportan las tecnologías, y sincronía en las comunicaciones entre los miembros (Kirkman y Mathieu, 2005). La mayoría de los estudios sobre virtualidad se han centrado en comparar equipos tradicionales con equipos virtuales (Martins, Gilson y Maynard, 2004), mostrando cómo la virtualidad modifica las interacciones entre los miembros del equipo. Trabajar de forma regular en condiciones de alta virtualidad limita las señales del contexto social presentes en la comunicación cara 
a cara, reduce la profundidad de la discusión y el análisis de alternativas, y aumenta el tiempo necesario para tomar decisiones colectivas. Diferentes estudios muestran que la virtualidad se relaciona con una comunicación menos eficiente y genera más dificultades para desarrollar confianza entre los miembros del equipo (Jarvenpaa y Leidner, 1999) o incrementar los niveles de apoderamiento (Kirkman, Rosen, Tesluk y Gibson, 2004).

De nuevo, los efectos de la virtualidad sobre los procesos y la eficacia del equipo dependen de las demandas de la tarea (Rico, Cohen y Gil, 2006), de forma que cuando los equipos realizan tareas complejas y/o interdependientes, resulta recomendable reducir los niveles de virtualidad (usando medios de comunicación síncronos y más ricos, como las videoconferencias o las reuniones cara a cara). No obstante, hay que considerar que los equipos se adaptan progresivamente a las condiciones de virtualidad impuestas por su trabajo, de manera que conforme aprenden a usar la tecnología para comunicarse y desarrollan nuevas estrategias para realizar sus tareas, los efectos de la virtualidad se debilitan (Lira, Ripoll, Peiró y González, 2007).

Liderazgo. Los últimos 10 años de investigación en este ámbito han pivotado sobre tres aspectos fundamentales: la consideración del liderazgo como algo externo al equipo, el coaching y el liderazgo compartido. El liderazgo externo encarna el paradigma tradicional en el estudio del liderazgo en equipos y se centra en la influencia de una figura externa al equipo responsable de su rendimiento. La asunción básica es que dicha figura genera las líneas de acción básica del equipo, coordina su actividad y lo vincula a otras unidades organizacionales. La investigación se ha centrado esencialmente en identificar cómo las acciones de los líderes externos facilitan o dificultan el rendimiento del equipo (Druskat y Kayes, 2000). En este sentido, este tipo de liderazgo es visto como un insumo que afecta a distintos procesos de equipo (aprendizaje, coordinación, gestión de problemas o el grado en que la información es compartida), estados emergentes (potencia del equipo o el compromiso) y su rendimiento (Chen, Kirkman, Kanfer, Allen y Rosen, 2007). Un meta-análisis reciente agrupa los comportamientos de liderazgo externo siguiendo la tradicional distinción tarea/relación y encuentra que tanto los comportamientos orientados a la relación (en particular, transformacionales y de consideración), como los orientados a la relación (iniciación de estructura y ampliación de los límites del equipo) se relacionaban positivamente con la efectividad percibida del equipo (Burke, Stagl, Klein, Goodwin, Salas y Halpin, 2006). Y el meta-análisis ya citado de Stewart (2006) mostraba cómo el liderazgo transformacional y el liderazgo que facilitaba el empoderamiento de los miembros incrementaban el rendimiento de equipo.

El coaching se define como la interacción directa con un equipo con la intención de ayudar a sus inte- grantes a realizar un uso coordinado y apropiado de sus recursos colectivos para realizar la labor del equipo (Hackman y Wageman, 2005), e incluye comportamientos orientados a la identificación de problemas en el equipo, la consulta sobre procesos o bien la identificación y compensación de la autogestión en el equipo. La literatura científica muestra efectos positivos de tales prácticas sobre la satisfacción o la seguridad psicológica de sus miembros (Edmonson, 1999; Wageman, 2001), pero no es concluyente con relación a su efecto sobre el rendimiento del equipo (Wageman, 2001). A este respecto, se han señalado distintas variables que podrían estar modulando el efecto del coaching sobre el rendimiento, como son los factores de diseño del equipo, especialmente la autonomía, o la estabilidad del entorno en el que se realiza la tarea (Wageman, 2001; Morgeson, 2005). Adicionalmente, el trabajo seminal de Hackman y Wageman (2005) desarrolla un modelo teórico de coaching que enfatiza, por un lado, su carácter funcional, en la medida en que desempeña funciones motivacionales, consultivas y formativas; y por otro, su carácter de desarrollo, en la medida en que las diferentes intervenciones se asociarían de forma más efectiva a distintos momentos en el desarrollo del equipo. Esta aproximación del liderazgo, aunque no es nueva, sí supone un área en la que la práctica y la investigación han de desarrollarse en un futuro.

Finalmente, el liderazgo compartido se refiere a una propiedad emergente en el equipo resultante de la distribución de las funciones de liderazgo entre múltiples miembros del equipo, en vez de estar concentradas en un único líder formal (Carson, Tesluk y Marrone, 2007). Este aspecto del liderazgo acumula escasa investigación. Los estudios se han centrado en identificar condiciones precursoras del liderazgo compartido entre las que se señalan el coaching (Carson et al., 2007), la orientación colectivista de los integrantes del equipo (Hiller, Day y Vance, 2006) o sus habilidades de influencia (Taggar, Hackett y Saha, 1999). La evidencia empírica disponible apoya mayoritariamente una influencia positiva del liderazgo compartido sobre el rendimiento del equipo (Carson et al., 2007; Ensley, Hmielesky y Pierce, 2006; Sivasubremaniam, Murry, Avolio y Jung, 2002). No obstante, existen resultados que ponen de manifiesto que el liderazgo distribuido no necesariamente ejerce un efecto positivo sobre el rendimiento del equipo (Mehra, Smith, Dixon y Robertson, 2006). Esto está generando la necesidad de avanzar hacia una conceptuación más completa del fenómeno de la mano de análisis de redes y la consideración de sus aspectos cíclicos, interactivos y temporales que subyacen a su emergencia y desarrollo (Carson et al., 2007; Day et al., 2004; Mehra, et al., 2006).

Composición del equipo. La composición se refiere a los atributos de los miembros del equipo y a su apropiada combinación para formar equipos efectivos. Dos aspectos de la composición del equipo han sido 
objeto de numerosos estudios: el tamaño (número de personas que forman el equipo) y las características de sus miembros. La cuestión central respecto al tamaño es conocer el tamaño óptimo del equipo. Conforme aumenta el tamaño de un equipo, aumenta la cantidad de recursos disponibles, pero también las necesidades de coordinación. Las investigaciones indican que el tamaño óptimo depende de ciertas contingencias. Por ejemplo, cuando la interdependencia requerida para realizar bien la tarea es alta y el ambiente externo inestable, resulta recomendable crear equipos pequeños (Kozlowski y Bell, 2003). No obstante, en un reciente estudio realizado con 329 grupos de trabajo que realizaban diferentes tipos de tareas en diversas organizaciones, Wheelan (2009) ha encontrado de manera sistemática que los equipos formados por entre 3 y 6 miembros eran significativamente más productivos y se encontraban más desarrollados que los compuestos por entre 7 y 10 y que los constituidos por más de 11 miembros, lo que parece indicar que el tamaño pequeño es preferible a cualquier otro.

Otro aspecto identificado de suma importancia es el cambio de las características de los miembros que componen el equipo. Las publicaciones al respecto se incrementan año a año por el impacto que el cambio tiene en los equipos contemporáneos y por el papel que el aumento o reducción del número de miembros y la integración de nuevos miembros tiene para explicar la adaptabilidad de los mismos (DeRue, Hollenbeck, Johnson, Ilgen y Jundt, 2008). Modificar la composición de un equipo puede perjudicar su eficacia. Pero, si los cambios sólo afectan a un número reducido de miembros y se realizan gradualmente, el rendimiento (innovación) puede mantenerse o incluso mejorar (Perreti y Negro, 2007). Relacionado con el cambio, el tiempo que los miembros trabajan juntos como equipo es también un factor relevante tanto para el desarrollo de los modelos mentales del equipo como para la coordinación. Así, los miembros del equipo que más tiempo pasan juntos adquieren un conocimiento común preciso sobre las habilidades que posee cada uno para el trabajo (memoria transactiva), lo que les ayuda a coordinar mejor sus acciones (Lewis, Belliveau, Herndon y Keller, 2007).

Respecto a las características de los miembros, un tópico de gran interés en la última década es la diversidad en la composición del equipo. La diversidad en equipos se ha identificado como un elemento facilitador de los procesos de innovación, toma de decisiones y solución de problemas (Tjosvold, Hui, Ding y $\mathrm{Hu}$, 2003). Pero distintas revisiones recientes nos muestran un panorama de resultados mixtos, que nos impide afirmar categóricamente que la diversidad conduce a mayores niveles de rendimiento o mejora el compromiso y la satisfacción de sus integrantes (Jackson y Joshi, 2004; Williams y O’Reilly, 1998). Esto ha producido la emergencia de aproximaciones como el estudio de las fallas (Lau y Murninghan, 1998), en las que lo relevante no es la distribución o el promedio de características individuales en un equipo, sino cómo dichas variables se alinean e interactúan entre sí. Las fallas se definen como líneas hipotéticas que dividirían a un grupo en subgrupos en función del alineamiento de uno o más atributos.

Es la investigación sobre fallas la que está guiando la investigación en diversidad en los últimos años, centrándola en tres aspectos principales: primero, en mostrar una relación curvilínea (U invertida) entre el potencial de falla en un equipo y su rendimiento (Gibson y Vermeulen, 2005); segundo, en identificar aspectos de tarea que modulan el impacto de las fallas (Rico, Molleman, Sánchez-Manzanares y van der Vegt, 2007); y tercero, en identificar aquellos mecanismos mediadores, como el conflicto, la identificación grupal (Lau y Murninghan, 2005) o el procesamiento de información relevante para la tarea, que expliquen los efectos de las fallas de diversidad (Homans y van Knippenberg, 2006).

Como conclusión de este ámbito de investigación podemos afirmar que sólo los niveles moderados de potencial de falla en un equipo facilitan el logro de mayores niveles de rendimiento (Gibson y Vermeulen, 2006). El equipo en estas circunstancias es suficientemente diferente para aprovechar la diversidad existente y suficientemente similar para que el conflicto no impida su funcionamiento. Niveles bajos o altos de potencial de falla plantearán obstáculos para el desempeño del equipo, por razones bien distintas: en el primer caso porque la similitud entre los miembros del equipo dificultará la existencia de información o perspectivas diversas; mientras que en el segundo, se precisará de más tiempo para integrar conocimientos y puntos de vista diferentes y la formación de subgrupos enfrentados dentro del equipo elevará el nivel de conflicto.

\section{Análisis DAFO de los hallazgos relativos a los insumos}

A lo largo de la sección anterior hemos resumido los principales hallazgos de investigación relativos a los insumos de los equipos de trabajo llevados a cabo en la última década. En esta sección discutiremos brevemente las principales debilidades, amenazas, fortalezas y oportunidades que, a nuestro juicio, presenta la investigación de los insumos en los equipos de trabajo. La Tabla 1 recoge el resultado de dicho análisis DAFO de forma abreviada.

Debilidades. De forma sintética podríamos agrupar en cuatro aspectos las debilidades que identificamos en el estudio de los insumos. El primero tiene que ver con la constatación de que el estudio sistemático de los equipos de trabajo requiere un notable esfuerzo (Mathieu, et al., 2008). Un esfuerzo que no tiene tanto que ver con el tamaño muestral, que también, sino 
fundamentalmente con la obtención de niveles de variabilidad suficiente para poder testar nuestras hipótesis.

El segundo, se plantea cuando comprobamos que la mayoría de los estudios mantienen una definición unitaria de los insumos a nivel de equipo frecuentemente, por ejemplo a la hora de medir y tratar la autonomía, la interdependencia o la virtualidad. En este contexto ¿cómo describiríamos un caso en el que algunos miembros trabajan unos con otros con un elevado nivel de interdependencia, mientras que con otros mantienen un nivel de interdependencia muy bajo? Estas constataciones han de hacernos pensar en alternativas de medida más refinadas y apropiadas, por ejemplo, asimetrías en interdependencia (de Jong et al., 2007).

Cuanto acabamos de decir para las características observamos esto (Katz, Lazer, Arrow y Contractor, 2004)

Finalmente, por la misma razón que los aspectos que facilitan el rendimiento no se trasladan de manera directa a distintos tipos de equipos de forma equivalente (Cohen y Bailey, 1997), no está claro si ciertos comportamientos asociados con el rendimiento en equipos tradicionales se mantienen a través de distintos grados de virtualidad (Maruping y Awargal, 2004). Una muestra de cuanto decimos lo ilustra el grado en que el estudio del liderazgo virtual es atendido, pero los resultados no muestran que sea bien entendido. Quizá tengamos que considerar que la teoría que conocemos relativa a los comportamientos de liderazgo no pueda ser necesaria y directamente aplicada sobre equipos que presentan niveles elevados de virtualidad.

Tabla 1. DAFO insumos

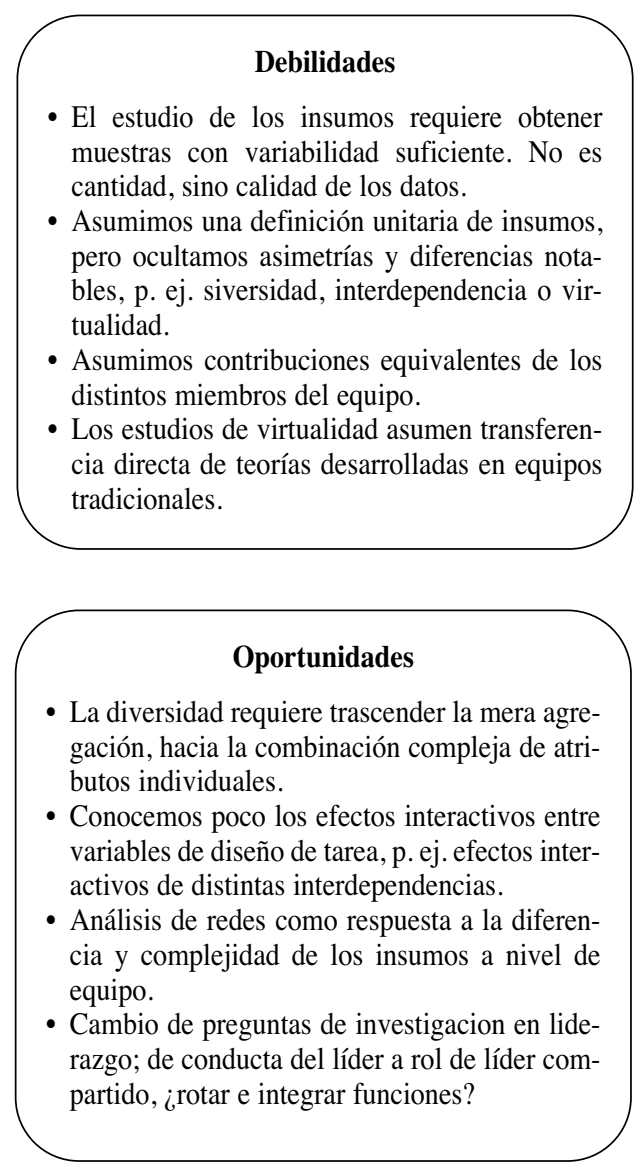

del diseño de la tarea se aplica con más fuerza, si cabe, sobre los estudios de diversidad. Bajo un indicador de heterogeneidad unitario se esconden dos trampas: a) ¿qué significa realmente un promedio o varianza de características individuales representadas en un equipo? (Lau y Murninghan, 1998), y b) asumimos una contribución equivalente de los distintos miembros del equipo a sus tareas y objetivos cuando en realidad no

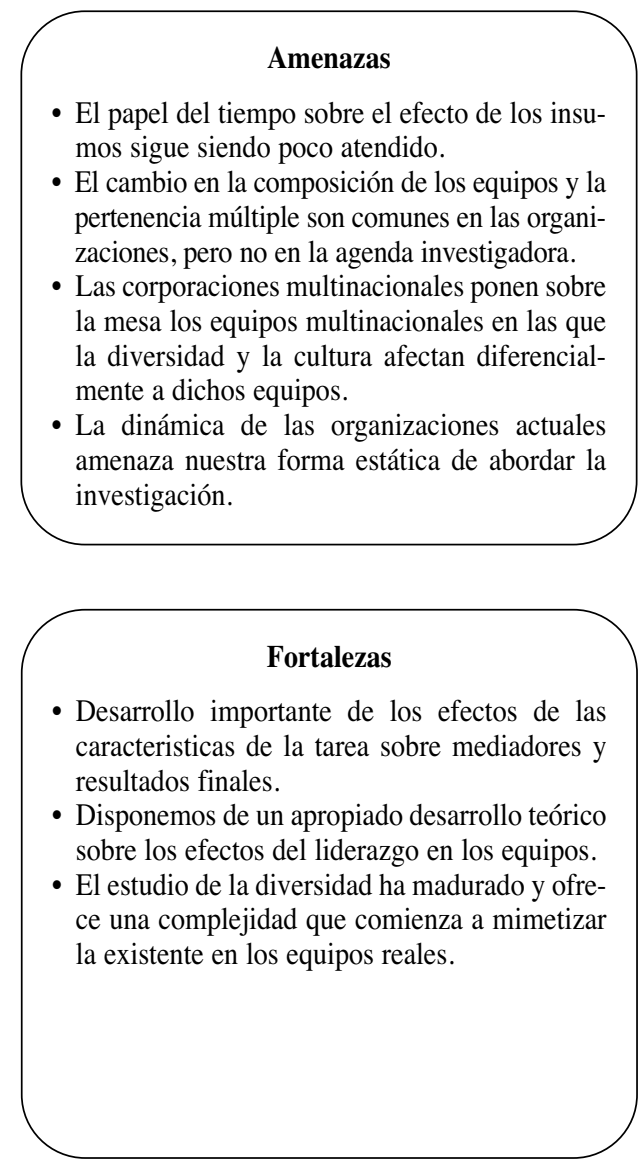

Amenazas. La principal amenaza que detectamos en este ámbito de investigación tiene que ver con la aparente incapacidad de los estudios actuales para dar cuenta de la complejidad inherente al estudio de los equipos. En este sentido, el papel del tiempo y el cambio en la composición de los equipos son aspectos escasamente atendidos. Esto se hace aún más saliente por la temporalidad inherente de muchos equipos de 
proyecto y porque la pertenencia múltiple a distintos equipos es algo común. Esta es una realidad que claramente amenaza nuestra forma estática, lineal y unidireccional de estudio de los equipos de trabajo. Honestamente, hemos de atender esta realidad con otro tipo de diseños longitudinales que consideren las dinámicas no lineales como estrategia de análisis del cambio.

Otro aspecto que planea como amenaza tiene que ver con el hecho de que las corporaciones multinacionales ponen sobre la mesa los equipos multinacionales en las que la diversidad y la cultura afectan diferencialmente a dichos equipos (Gibson, 2003). Sorprende, sin embargo, la escasa atención investigadora sobre la influencia cultural en los equipos de trabajo.

Fortalezas. El estudio de los insumos en los equipos de trabajo ha experimentado un notable desarrollo que nos permite comprender mejor los efectos de las características de la tarea sobre mediadores y resultados finales (Mathieu et al., 2008). También disponemos de un apropiado desarrollo teórico sobre los efectos del liderazgo en equipos, que nos permite observar con claridad el camino hacia el liderazgo distribuido y al cambio de las preguntas de investigación al respecto. Dotarnos de muestras con suficientes niveles de variabilidad es posible, disponemos de opciones, como el muestreo selectivo o los estudios de macro-colaboración tipo Globe Project (House, Javidan, Hanges y Dorfman, 2002).

Así mismo, tras 40 años de acumulación de evidencias contradictorias, el estudio de la diversidad madura y ofrece alternativas que aúnan complejidad y facilidad de comprensión, de modo que nos permiten un tratamiento más ecológico de la diversidad en los equipos (van Knippenberg y Schippers, 2007).

Oportunidades. Trascender nuestras debilidades y convertir nuestras amenazas en oportunidades implica el estudio de los equipos de trabajo siendo sensibles a su complejidad. En el caso de la diversidad precisamos movernos más allá de la mera agregación de atributos individuales y plantearnos la investigación de diversidad como una combinación compleja de atributos individuales (Lau y Murninghan, 2005). A ello ayudará el desarrollo futuro del paradigma de las fallas de diversidad.

Así mismo, sabemos muy poco de los efectos interactivos entre variables del diseño de la tarea, apenas existen trabajos, por ejemplo, sobre los efectos interactivos de los distintos tipos de interdependencia (hipótesis de la congruencia) o los efectos interactivos de la autonomía y la interdependencia (Langfred, 2005). Adicionalmente, el aprovechamiento de medidas más sensibles a las asimetrías y centralidades, como las que nos ofrece el análisis de redes (Scott, 2000), nos acercan a estudios que respondan a las diferencias y complejidades de los insumos a nivel de equipo.

Finalmente, sorprende el volumen de literatura dedicado al liderazgo y lo poco que sabemos sobre cómo los líderes crean y gestionan grupos de trabajo (Zaccaro, Rittman y Marks, 2001). Quizá la investigación necesita trascender el debate de si es mejor el liderazgo externo frente al basado en los miembros del equipo y plantearse el liderazgo como un conjunto de funciones que necesitan cumplimentarse y satisfacerse en los equipos (Mathieu et al., 2008; Day et al., 2004). Con ello es muy probable que se produzca un giro en las preguntas de investigación hacia la exploración de la complejidad inherente al liderazgo distribuido.

\section{Mediadores: procesos, estados emergentes y mediadores mixtos}

Los mediadores consisten en un conjunto de mecanismos psicosociales que permiten a los miembros de un equipo combinar los recursos disponibles para realizar el trabajo asignado por la organización, superando las dificultades derivadas de la coordinación y la motivación de sus integrantes. El estudio de los mediadores es un área que está llamada a evolucionar de manera importante en los próximos años. La investigación se acumula y a pesar de la actual diferenciación entre procesos, estados emergentes y mediadores mixtos (Mathieu et al., 2008), la literatura no acaba de contar con una agrupación consensuada relativa a los mediadores.

Procesos. Los procesos han jugado un papel central en los modelos de efectividad grupal más populares (Guzzo y Dickson, 1996; Hackman, 1983). Tradicionalmente se han dividido en procesos de tarea, que dan cuenta de las funciones que las personas deben realizar para realizar satisfactoriamente la tarea del equipo, y en procesos de relación, que describen la interacción entre los miembros del equipo (McIntyre y Salas, 1995). Basándose en esta distinción y en una aproximación multifase de los procesos Marks et al. (2001) proponen una taxonomía de procesos de equipo que identifica tres categorías: transición, acción e interpersonales. Vamos a utilizar dicha diferenciación para revisar el estado actual de la literatura sobre procesos.

Los procesos de transición permiten al equipo preparar el escenario para la acción futura. Los esfuerzos investigadores en esta área se han centrado fundamentalmente sobre la generación de estrategias y planificación. En general, la evidencia empírica muestra cómo distintos aspectos relativos a la planificación y la elección de una estrategia, como son la deliberación previa de planes, la anticipación de posibles problemas o la cantidad de información compartida y las oportunidades de participación ofrecidas a los miembros del equipo correlacionaban positivamente con los niveles de efectividad del equipo (Janicik y Bartel, 2003; Mathieu y Rapp, 2009; Mathieu y Schulze, 2006; Pritchard, Jones, Roth, Stuebing y Ekeberg, 2001; Tesluk y Mathieu, 1999). 
Con relación a los procesos de acción, la investigación se ha centrado esencialmente en comportamientos de ayuda y reparto de la carga de trabajo, así como de coordinación y comunicación. La relación entre los procesos de coordinación y comunicación y el rendimiento del equipo ha recibido considerable soporte empírico (LePine, 2003; LePine et al., 2008; SánchezManzanares, Rico, Gibson y Kearney, en revisión; Tesluk y Mathieu, 1999). El efecto positivo de las actividades de coordinación resulta especialmente significativo cuando los miembros se enfrentan a estrés por sobrecarga de trabajo (Rothrock, Cohen, Yin, Thiruvengada y Nahum-Shani, 2009). Adicionalmente, los estudios centrados en el desarrollo de la monitorización y reparto del trabajo han mostrado que dichos comportamientos predicen el rendimiento en los equipos (De Dreu y West, 2001; Porter, 2005), en tanto que la holgazanería social amenaza el cumplimiento de objetivos del equipo (Høigaard, Säfvenbom y Tønnessen, 2006).

Finalmente, respecto a los procesos interpersonales, es el conflicto el que acapara gran parte de la atención investigadora en esta última década. Existe un claro consenso en las consecuencias negativas que el conflicto de relación ejerce sobre el rendimiento (De Dreu y Weingart, 2003), mientras que los efectos del conflicto de tarea sobre el rendimiento no son del todo concluyentes (Jehn, Northcraft y Neale, 1999) y sugieren una relación curvilínea y más compleja con el rendimiento que incluye el concurso de otros estados emergentes como la potencia o la confianza (Gil, Rico y Sánchez-Manzanares, 2008).

Estados emergentes. Se definen como estados cognitivos, motivacionales o afectivos del equipo, de carácter dinámico y variable en función del contexto, insumos, procesos y resultados del equipo (Marks et al., 2001). En este sentido, los estudios sobre potencia y "team efficacy", el clima de equipo, la cohesión, la confianza o la cognición compartida, han configurado el grueso de la investigación sobre estados emergentes.

La potencia y la "team efficacy" han sido tratadas de forma conjunta, a pesar de que la primera se refiere a una creencia colectiva relativa a la capacidad del equipo para ser efectivo y la segunda concierne dicha creencia aplicada a tareas y contextos particulares (Kozlowski e Ilgen, 2006; Shea y Guzzo, 1987). En un meta-análisis relativamente reciente se pone de manifiesto cómo ambas variables mantienen una relación moderadamente positiva con el rendimiento, sobre los que la interdependencia del equipo ejercen una modulación positiva y aumentativa (Gully, Incalcaterra, Joshi y Beaubien, 2002).Otro estudio reciente (Tasa, Taggar y Seijts, 2007) muestra apoyo a cómo la eficacia colectiva (o de equipo), junto con la autoeficacia para el trabajo en equipo y el conocimiento relevante para la tarea predicen las conductas de equipo de los miembros, y a su vez, éstas incrementan la percepción de eficacia colectiva, que contribuye significativamen- te al rendimiento final del equipo. Estos resultados proporcionan apoyo a la tesis defendida por Marks et al. (2001), según la cual los estados emergentes no son procesos grupales en sí mismos.

La identidad de equipo, definida como el grado en que una persona se define a sí misma como miembro de un equipo particular. Este estado emergente ha sido propuesto como una variable mediadora entre la diversidad del equipo y la reducción voluntaria de los esfuerzos hacia el grupo (ej. holgazanería social), especialmente en entornos virtuales de trabajo (Shapiro, Furst, Spreitzer y von Glinow, 2002). Resultados recientes corroboran cómo la identidad de equipo orienta a sus miembros hacia el conjunto del grupo, limitando comportamientos improductivos y generando un mayor número de conductas cooperativas que los miembros con menores niveles de identificación con su equipo (Eckel y Grossman, 2004).

El clima de equipo se define como el conjunto de normas, actitudes y expectativas percibidas que operan en un contexto social determinado (Pirola-Merlo et al., 2002). Su estudio en la última década se ha refinado, de la mano de conceptos como la fortaleza de clima (González-Romá, Peiró y Tordera, 2002) y del florecimiento de dimensiones específicas de clima, como el clima para la innovación, el clima de seguridad o el clima de justicia. Con respecto al clima de innovación (Anderson y West, 1998), la dimensión de soporte para la innovación ha sido el predictor más consistente de la innovación de equipo evaluada externamente (Burningham y West, 1995). El clima de seguridad ha ofrecido un nivel predictivo significativo respecto al nivel de accidentes del equipo (Zohar, 2000) y también desde una perspectiva multinivel prediciendo cambios a nivel individual relativos a la motivación por la seguridad (Neal y Griffin, 2006). Finalmente, el clima de justicia se relaciona significativamente, de manera directa con el rendimiento del equipo, y de manera inversa con el absentismo (Colquitt, Noe y Jackson, 2002) y ejerce también efectos multinivel que predicen el compromiso con la organización a nivel individual (Yang, Mossholder y Peng, 2007).

La cohesión definida como el compromiso de los miembros de un equipo entre sí, o con la tarea a realizar, ha sido uno de los estados emergentes más estudiados. Prueba de ello son los recientes meta-análisis efectuados, como el de Beal, Cohen, Burke y McLendon (2003) en el que se examinaron estudios llevados a cabo en los últimos 50 años, mostrando una correlación media corregida entre cohesión con rendimiento general y comportamientos de rendimiento de .17 y .30 respectivamente. No obstante estos resultados globales, otro meta-análisis reciente muestra que el tipo de equipo y el tipo de contexto organizacional influyen en el nivel de cohesión y en el subsecuente rendimiento, encontrando que los equipos de proyecto que trabajan en contextos organizacionales y académicos muestran mayor tamaño del efecto que en el caso 
de los equipos de producción y en los equipos de servicio (Chiochio y Essiembre, 2009). Por otra parte, la cohesión también ha sido estudiada como mediador entre la relación de liderazgo transformacional y rendimiento (Bass, Avolio, Jung y Berson, 2003).

La confianza es otro de los estados emergentes más investigados en los últimos años. Definida como la propensión de una persona a ser vulnerable a las acciones de otra, basada en la expectativa de que dicha parte lleve a cabo una acción particular relevante para quien confía, con independencia de su habilidad para controlar las acciones de la persona confiada (Mayer, Davis y Schoorman, 1995). Los esfuerzos se han centrado en revelar su carácter modulador sobre distintos insumos organizacionales, como el beneficio de la formación o los niveles de autonomía del equipo, sobre el rendimiento (Kirkman, Rosen, Tesluk y Gibson, 2004; Langfred, 2005). Así mismo, de la mano del estudio de la virtualidad ha existido un interés importante en el estudio de su desarrollo (Jarvenpaa y Leidner, 1999; Jarvenpaa, Shaw y Staples, 2004, Wilson, Strauss y McEvily, 2006) y del papel modulador que sobre su desarrollo ejerce la interdependencia de la tarea (Rico, Alcover, Sánchez-Manzanares y Gil, 2009).

La cognición colectiva ha sido otra de las áreas que ha arrastrado un importante volumen de investigación (Mathieu et al., 2008), destacable especialmente en el ámbito de los modelos mentales compartidos (MMC). Los MMC se definen como la representación mental del conocimiento que es compartido por los miembros de un equipo (Mathieu et al., 2005). Son clasificados en dos tipos principales, MMC de tarea y de equipo cuyos efectos positivos sobre el rendimiento son indirectos y directos respectivamente. Así mismo, estudios de campo han mostrado el efecto interactivo que se produce entre los dos tipos de MMC sobre la seguridad en el trabajo y la eficiencia (Smith-Jentsch, Mathieu y Kraiger, 2005). La evolución reciente del estudio de los MMC ha revelado la importancia de una diferenciación ulterior, como es el grado en que los MMC son compartidos y precisos (Lim y Klein, 2006). A este respecto, trabajos recientes muestran la mayor capacidad predictiva sobre el rendimiento de la precisión frente al grado en que son compartidos los MMC (DeChurch y Mesmer-Magnus, en prensa; Edwards, Day, Arthur y Bell, 2006). Finalmente los MMC han sido postulados como un elemento clave para entender los mecanismos mediante los que tiene lugar la coordinación tanto implícita como explícita en los equipos de trabajo (Rico, Gibson, Sánchez-Man-zanares y Clark, 2009; Rico, Sánchez-Manzanares, Gil y Gibson, 2008)

Mediadores mixtos. Existen un conjunto de mediadores que no son fácilmente clasificables en las dos categorías anteriores, dado que suponen una mezcla entre procesos y estados emergentes, en nuestra revisión vamos a considerar dos: el aprendizaje y la memoria transactiva.
El aprendizaje de equipo representa un proceso continuo de reflexión y acción mediante el que los equipos adquieren, comparten, combinan y aplican conocimiento (Edmonson, 1999). La literatura sobre aprendizaje de equipo ha mostrado su relación positiva con la calidad de las relaciones entre los miembros del equipo y con el rendimiento (Zellmer-Bruhn y Gibson, 2006). Así mismo, ha mostrado su papel mediador entre la seguridad psicológica y el rendimiento (Edmonson, 1999) y el grado en que se facilita mediante mecanismos de coaching (Hackman y Wageman, 2005).

Los sistemas de memoria transactiva (SMT), se componen de los conocimientos y habilidades que aportan los diferentes miembros del equipo, así como del conocimiento que poseen sobre la distribución de dichos conocimientos y habilidades entre ellos (quién sabe qué en el equipo). Trabajos recientes revelan que los equipos que se comunican más frecuentemente generan SMT más potentes (Lewis, 2004; Yoo y Kanawattanachai, 2001), que se relacionan tanto con el rendimiento como con la viabilidad del equipo (Lewis, 2004). El constructo se ha desarrollado de forma que, al igual que en el caso de los MMC, se considera que existen distintos tipos de SMT, relativos a la tarea y a las relaciones externas del equipo (Austin, 2003). Finalmente, desarrollos recientes extienden el papel de los SMT a la transferencia de aprendizajes previos de los miembros del equipo (Lewis, Lange y Gillis, 2005) y muestran el papel mediador que juega en la relación entre la sobrecarga que la tarea ejerce sobre el equipo y su rendimiento, así como entre la comunicación funcional y el rendimiento y la satisfacción (Ellis, 2006; Sánchez-Manzanares et al., 2006).

\section{Análisis DAFO de los hallazgos relativos a los mediadores}

La sección precedente da cuenta de los principales resultados de investigación concernientes a los mecanismos mediadores de la efectividad los equipos de trabajo ocurridos en la última década. En este apartado analizaremos brevemente las principales debilidades, amenazas, fortalezas y oportunidades de la investigación con relación a los mecanismos mediadores en los equipos de trabajo. La Tabla 2 ofrece de manera sumaria el resultado del análisis DAFO efectuado.

Debilidades. La investigación de procesos de equipo es fragmentada, tan sólo contamos con dos trabajos que han propuesto y fundamentado un marco integrador de los mismos (Marks et al., 2001 y LePine et al., 2008) y apenas existen tres artículos que consideran de manera conjunta más de una dimensión global de procesos (Mathieu et al., 2008). Una de las debilidades más notables es la alta correlación entre los procesos de equipo, entre los emergentes, y entre éstos y los primeros, especialmente cuando se miden al mismo tiempo y utilizando los mismos informantes (LePine et al., 


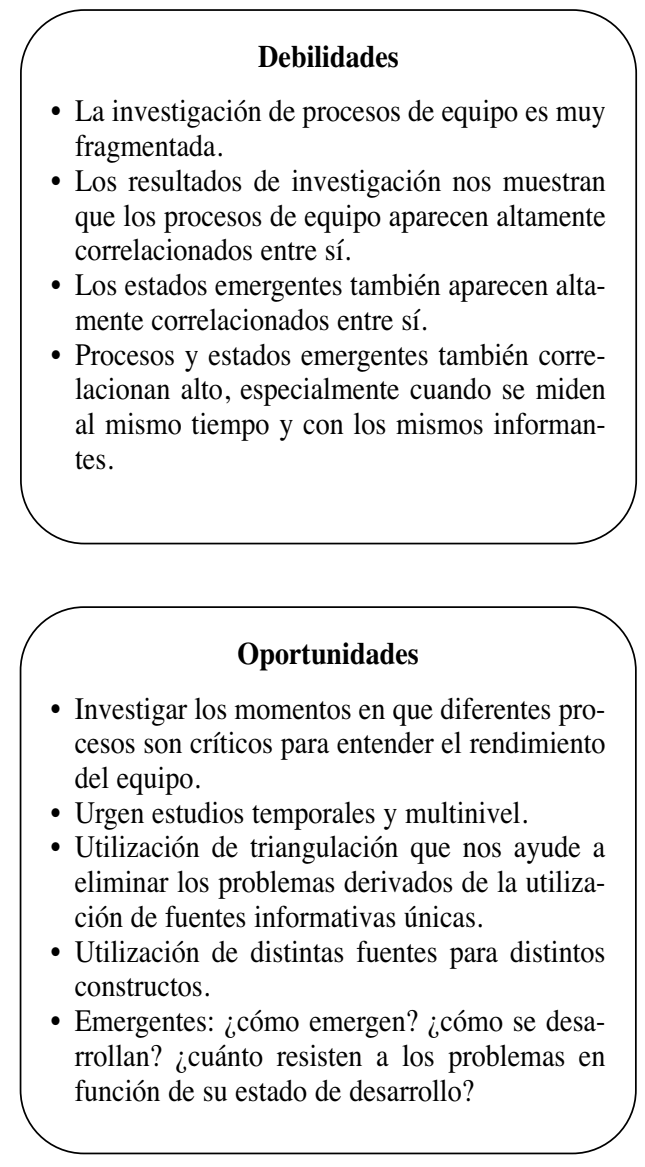

2008). Todo esto ofrece un panorama que debe hacernos reflexionar sobre las técnicas y momentos para la medida de los diferentes procesos.

Amenazas. Las multicolinealidad amenaza seriamente la validez de los resultados derivados de los estudios de los distintos mediadores, al menos en tres formas distintas: primero, la existencia de mediadores que funcionen como variables enmascaradas y por tanto, que nos impidan saber si el efecto de un proceso se debe a otro proceso distinto, que no estemos controlando en nuestros análisis; segundo, desconocemos si los informantes son capaces de establecer y reconocer la diferenciación entre los distintos procesos sobre los que son inquiridos; y tercero, las altas correlaciones entre los mediadores revelan de forma inequívoca nuestra dificultad para aplicar diseños temporales que nos permitan medir estratégicamente los mediadores en el momento que realmente afectan a los resultados (LePine et al., 2008).

Fortalezas. Afortunadamente, disponemos de modelos que ofrecen un marco integrador a los procesos de equipo y de modelos de fases de evolución (Kozlowski y Bell, 2008; Marks et al., 2001), que nos pueden guiar y dar un mayor sentido temporal a la medida de los mediadores de equipo. Así mismo, cono-

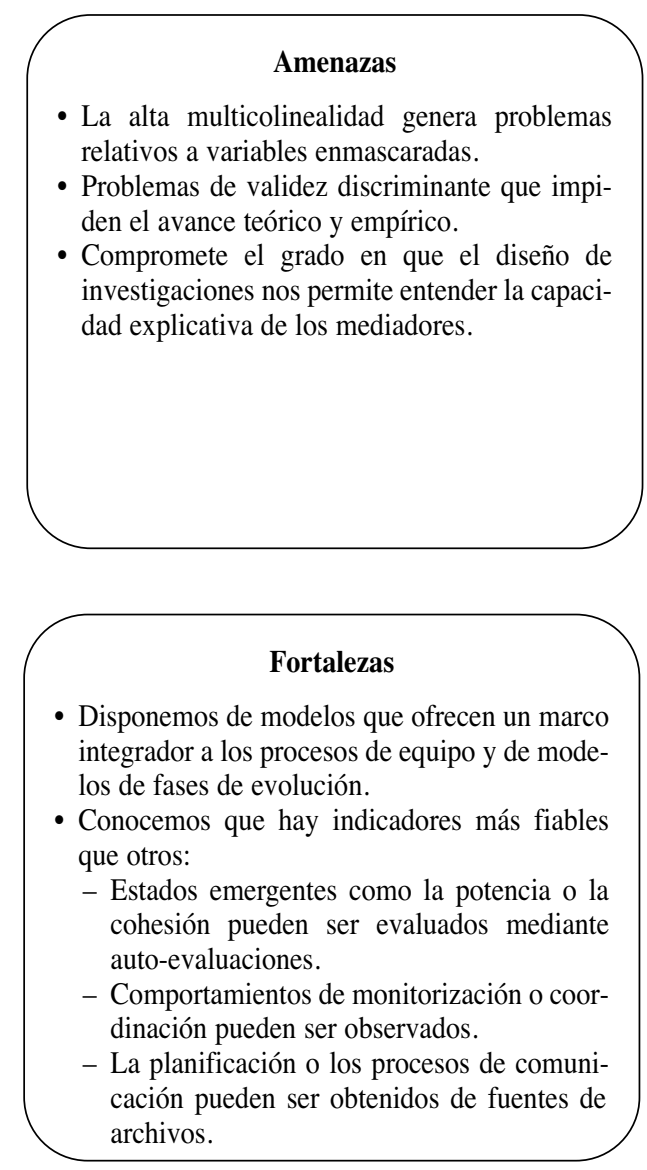

cemos que hay indicadores más fiables que otros a la hora de evaluar mediadores (Tesluk et al., 1997), por ejemplo, los emergentes como la potencia o la cohesión pueden ser evaluados mediante auto-evaluaciones, los comportamientos de monitorización o coordinación pueden ser identificados a partir de la observación del funcionamiento habitual del equipo; y la planificación o los procesos de comunicación pueden ser obtenidos de fuentes de archivos que registran la actividad del equipo.

Oportunidades. Investigar los momentos en que diferentes procesos y estados emergentes son críticos para entender el rendimiento del equipo supone una clara oportunidad para el desarrollo de la investigación y el diseño de soluciones aplicadas que respondan a las demandas de los equipos de trabajo. Además de incorporar diseños temporales en nuestras propuestas de investigación, es precisa la utilización de técnicas de medida basadas en la triangulación de indicadores que minimicen los problemas derivados de la utilización de una única fuente informativa y maximicen la validez discriminante y predictiva de los distintos mediadores.

Finalmente, la adopción de una mayor sensibilidad a los aspectos temporales requiere avanzar en el estudio del surgimiento y desarrollo de los estados emergen- 
tes. Si los emergentes no se desarrollan linealmente ¿cabe pensar en puntos de inflexión?; si los emergentes emergen, ¿cómo lo hacen?, ¿cómo emerge la confianza?, ¿cómo se desarrolla?, ¿cuánto resiste a las violaciones en función del momento de desarrollo del equipo?

\section{Resultados de equipo}

Los resultados alcanzados por el equipo son usualmente considerados desde una perspectiva multidimensional y multinivel (Mathieu et al., 2008). Generalmente, la eficacia se analiza en términos de resultados de trabajo, como rendimiento objetivamente evaluado (usando indicadores específicos o personal experto externo al equipo). Pero también incluye resultados que ayudan a mantener el desempeño del equipo a lo largo del tiempo, como la satisfacción de sus miembros, la viabilidad (grado en que los miembros del equipo desean permanecer juntos) y la innovación (Gil, Rico y Sánchez-Manzanares, 2008). Es importante recalcar lo sistemática que ha sido la literatura de equipos en identificar variables antecedentes y mediadoras y lo poco que lo ha sido a la hora de dar cuenta de los resultados (Mathieu et al., 2008). Por ello, adoptamos una aproximación que resume los resultados de equipo en tres niveles: organizacional, de equipo, de rol y en tres tipos: desempeños (las acciones relevantes para conseguir resultados), rendimiento (compuestos por las consecuencias del desempeño) y actitudes, y proponemos una matriz de $3 \times 3$ para dar cuenta de la evolución de la literatura en este aspecto.

Resultados a nivel individual. A este respecto, podemos destacar dos categorías principales, aquellos que tienen que ver con los requerimientos del rol desempeñado por los integrantes del equipo y con aquellos que informan de sus reacciones afectivas. Respecto a los resultados basados en el desempeño de rol, trabajos recientes muestran cómo este indicador puede ser utilizado satisfactoriamente para medir el rendimiento individual y compararlo entre distintos equipos (Chen, 2005; Chen et al., 2007). Con relación a las reacciones afectivas de los integrantes del equipo la satisfacción con el equipo, el trabajo y la organización, junto al compromiso organizacional son los indicadores que más atención han recabado (Kirkman y Rosen, 1999; Tesluk y Mathieu, 1999).

Resultados a nivel de equipo. Con relación a estos resultados se ha diferenciado, por un lado, entre desempeños y resultados (Beal, Cohen, Burke y McLendon, 2003); y por otro, la viabilidad de equipo (Balkundi y Harrison, 2006). Si nos referimos al desempeño destacan aquellos relacionados con la mejora de los procesos, con el aprendizaje y el rendimiento cognitivo. Por ejemplo, se evalúa la mejora de procesos midiendo la búsqueda de retroalimentación, la discusión de errores o la experimentación (Kirkman et al., 2006), el procesamiento de la información relevante para la tarea
(Homan et al., 2006), el aprendizaje (Edmonson, 1999), la calidad de las decisiones (Jehn y Shah, 1997) o la proactividad (Kirkman y Rosen, 1999).

Considerando el rendimiento de equipo las medidas utilizadas son notablemente diversas. Desde evaluaciones externas de clientes relativas a la calidad de servicio (Kirkman,Tesluk y Rosen, 2004) o la evaluación de responsables respecto al rendimiento, la calidad o la innovación (Langfred, 2000; Mathieu, Gilson y Rudi, 2006; Tjosvold, Tang y West, 2004) a indicadores de archivo relativos a la innovación o producción (Mathieu et al., 2006; Perreti y Negro, 2007). Uno de los aspectos de rendimiento de equipo que más investigación ha generado en estos últimos años ha sido el estudio de la innovación, definida como la generación e implementación de nuevas ideas (West y Farr, 1990). Muy recientemente, Hülsheger, Anderson y Salgado (2009) han revisado meta-analíticamente los resultados de 30 años de investigación en innovación. El estudio analiza los insumos y los procesos de equipo como variables antecedentes y el efecto modulador de distintos aspectos metodológicos relativos a los estudios. En este sentido, son tres los hallazgos principales: a) la comunicación externa e interna, el soporte para la innovación, la orientación a la tarea y la cohesión son procesos de equipo relacionados de manera significativa y generalizable con innovación en equipo; b) la interdependencia de objetivos, la diversidad relevante para el puesto y el tamaño del equipo son insumos conducentes a la innovación del equipo, aunque con menor fuerza de relación que los procesos anteriormente citados; y c) los efectos principales descritos, tanto para procesos como para insumos, son modulados por el nivel de análisis en el que se efectúa la medida de las variables. En este sentido, las relaciones entre las variables de procesos de equipo e innovación son más fuertes si consideramos la innovación a nivel de equipo, frente a nivel individual. Adicionalmente, las relaciones son más fuertes si se emplean indicadores autoinformados de innovación, frente a indicadores objetivos o externos.

Definiendo la viabilidad como la capacidad del equipo para permanecer juntos en el futuro, comprobamos que sigue siendo un resultado popular a nivel de equipo, combinado adicionalmente con otras medidas afectivas y actitudinales (Balkundi y Harrison, 2006; Barrick, Bradley, Kristof-Brown y Colbert, 2007). No obstante, debido a su carácter autoinformado no suele aparecer como criterio primario en las investigaciones. Los problemas persisten cuando se ha evaluado la viabilidad externamente. En este caso presenta importantes carencias de validez discriminante al compararla con otras respuestas afectivas sobre el rendimiento del equipo (Mathieu et al., 2008).

Resultados a nivel organizacional. Finalmente, con respecto a los resultados a nivel organizacional, constatamos su muy reciente incorporación a la literatura de investigación sobre equipos, a pesar de que para los 
equipos de dirección este referente debería ser imprescindible. Adicionalmente, el creciente interés por los sistemas multi-equipo en las organizaciones hace que la necesidad de establecer modelos de compilación que nos muestren cómo los distintos resultados de equipo se combinan para producir beneficios organizacionales (Mathieu et al., 2008). Entre tanto, podemos referir cómo distintos mediadores de equipo, como la comunicación, la cohesión o el cumplimiento de objetivos en el equipo, afectan positivamente los ratios financieros, el rendimiento y los beneficios globales de la organización (Barrick et al., 2007; Bunderson y Sutcliffe, 2002; Srivastava, Bartol y Locke, 2006).

\section{Análisis DAFO de los hallazgos relativos a los resultados}

Finalmente analizamos las principales debilidades, amenazas, fortalezas y oportunidades que presenta este ámbito de investigación, concluyendo así el resumen de los principales hallazgos relativos a los resultados de la efectividad de los equipos de trabajo en la última década. La Tabla 3 recoge de manera resumida los resultados del análisis DAFO llevado a cabo.
Debilidades. Del conjunto de la investigación en equipos de trabajo el ámbito relativo a los resultados es el menos especificado y elaborado de todos. Las variables criterio autoinformadas se utilizan con profusión, pero es difícil justificar que disponiendo de indicadores objetivos del rendimiento en un equipo, confiemos dicha medida a la percepción de sus integrantes. Paradójicamente, puede constatarse una limitada presencia en la última década de medidas afectivas de resultado, lo cual, sin embargo, justificaría la utilización de medidas autoinformadas. Finalmente, relacionado con debilidades que han aparecido en los insumos y en los mediadores, seguimos sin entender bien los efectos de las dinámicas temporales en los resultados de los equipos. Desconocemos si el rendimiento medido un par de meses después de administrar un cuestionario para medir insumos o procesos, tiene el mismo sentido que medirlo un año después (Mathieu et al., 2008). Así mismo, a pesar de que en estudios experimentales y de campo obtengamos medidas repetidas, estas se realizan de forma ciega (Johnson, Hollenbeck, Humphrey, Ilgen, Jundt y Meyer, 2006) y con escasa justificación respecto a las dinámicas de la tarea del equipo.

Tabla 3. DAFO resultados

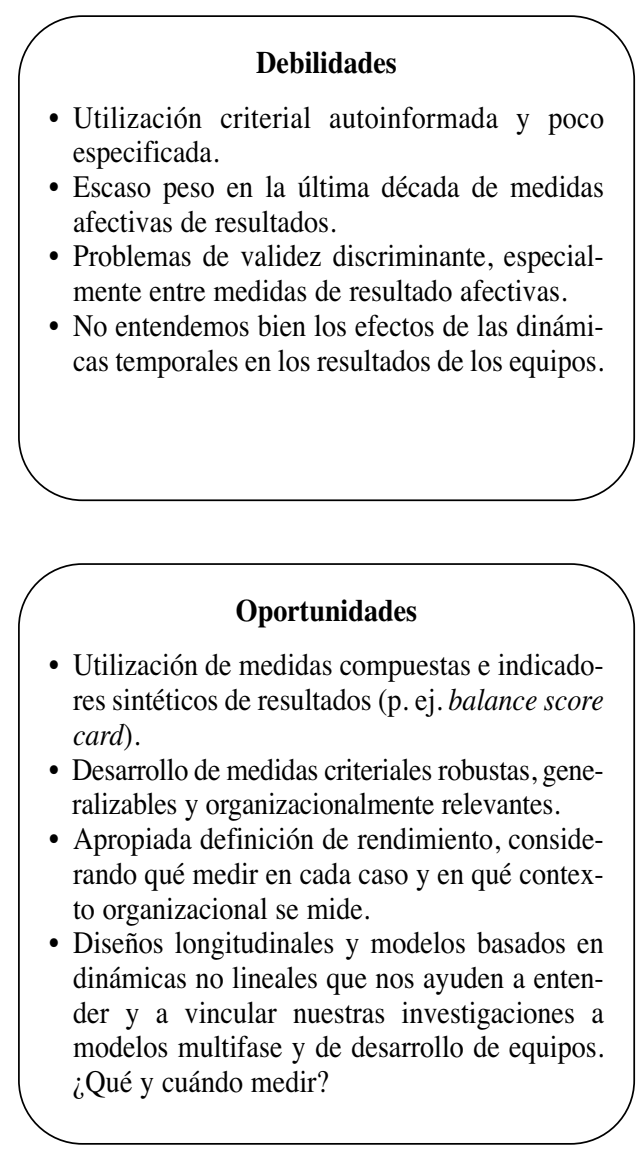

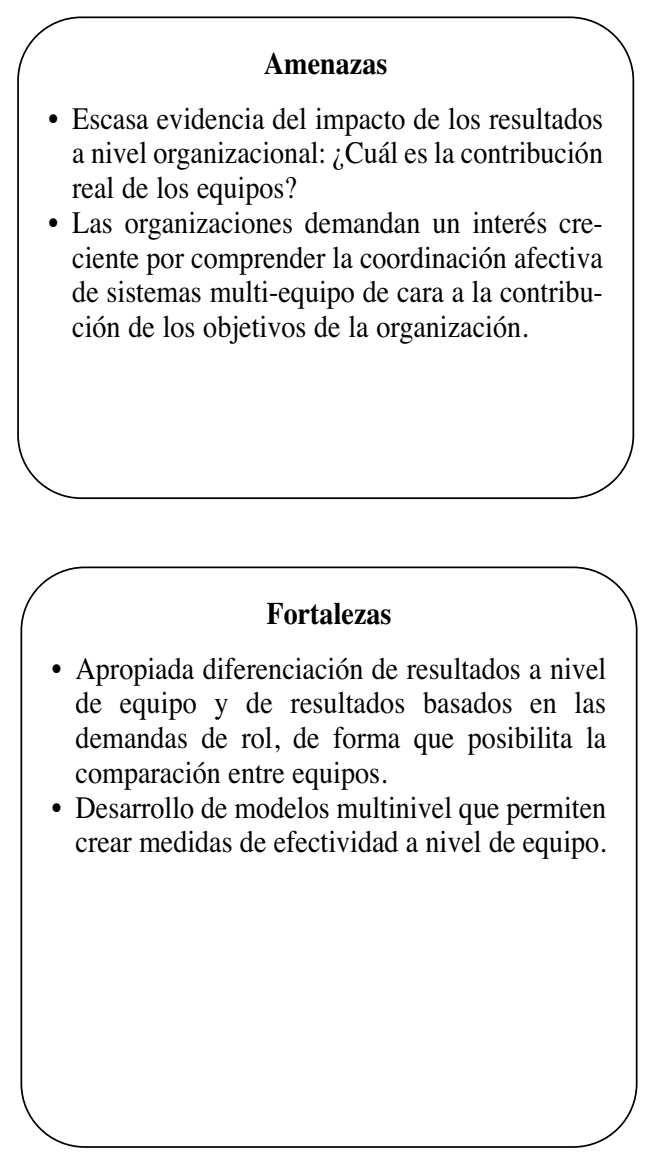


Amenazas. A pesar de los avances en los modelos multinivel, la literatura no ha desarrollado modelos que permitan comprobar cómo los procesos de equipo y sus resultados se combinan sobre los beneficios de la organización. En este sentido, una preocupación de fondo tiene que ver con conocer cuál es la contribución real de los equipos a la efectividad organizacional (Mathieu et al., 2008).

En línea con el anterior planteamiento, existe una preocupación creciente en las organizaciones por los sistemas multi-equipo (Hollenbeck, 2009) centrada esencialmente en disponer de un marco para entender la contribución a su efectividad del conjunto de equipos que las conforman (Rico y DeChurch, en revisión), bien sea en circunstancias excepcionales (equipos en situaciones de crisis o emergencias) o bien en circunstancias normales (constelación de equipos de diseño, producción y ventas).

Fortalezas. Dos son las fortalezas más importantes que nos ofrece el análisis de la literatura de equipos de la última década. Por un lado, la apropiada diferenciación de resultados a nivel de equipo y de resultados basados en las demandas de rol, que permiten la comparación entre equipos distintos en la misma y en diferentes organizaciones. Y por otro, el desarrollo y presencia que los modelos multinivel (Kozlowski y Klein, 2000) tienen en la literatura, permitiéndonos crear un buen número de medidas de efectividad a nivel de equipo.

Oportunidades. El reconocimiento de las múltiples funciones que desarrollan los equipos de trabajo debería inclinarnos hacia la utilización de medidas compuestas (tipo balanced scorecard) e indicadores sintéticos de resultados (Kirkman et al., 2004). De esta forma, avanzaremos en el desarrollo de criterios robustos, generalizables y organizacionalmente relevantes, al estilo de la propuesta ProMES de Pritchard (1995) que vinculaba de manera ponderada las funciones múltiples de los equipos y su relevancia organizacional.

Finalmente, el estudio de los resultados puede beneficiarse también del diseño de modelos basados en dinámicas no lineales que nos ayuden a entender y vincular nuestras investigaciones a modelos multifase y de desarrollo de equipos. No tiene el mismo sentido medir el grado en que un equipo innova en fases tempranas del desarrollo de un producto, que en fases tardías (Gilson y Shalley, 2004). Por tanto, una vez más, la cuestión radica en la apropiada definición de qué, cuándo y en qué contexto organizacional se realiza la medida.

\section{Discusión}

Los cambios estratégicos, económicos y tecnológicos reestructuran las organizaciones en torno a los equipos como unidades básicas de trabajo. Su eficacia y la comprensión de los desafíos que los sistemas multi-equipo plantean, constituyen factores clave para el éxito de las organizaciones contemporáneas.

Los enfoques teóricos actuales coinciden en considerar a los equipos como sistemas adaptativos complejos, cuya eficacia depende de la acción interactiva entre distintos insumos, procesos, estados emergentes derivados, mediadores mixtos y resultados. Pero los estudios en el ámbito de los equipos de trabajo se han centrado principalmente sobre insumos y resultados, aun cuando en este último caso, el esfuerzo de desarrollo sea menor. A pesar de que, en proporción, el estudio de los mediadores ha tenido menos peso, se observa en los últimos años un notable incremento del interés por los procesos y estados emergentes. En este sentido, se ha avanzado de manera decisiva en el estudio de la cognición de equipo, aunque se precisa más investigación para identificar los antecedentes, efectos, métodos de evaluación y mejora de dichos procesos cognitivos. Respecto a los estados emergentes emocionales, su desarrollo teórico es aún precario y son pocas las investigaciones que analizan sus efectos sobre la eficacia de los equipos. Finalmente, pese a que se considera fundamental investigar el funcionamiento del equipo a lo largo del tiempo, los estudios longitudinales o centrados en aspectos dinámicos de pertenencia al equipo siguen siendo la excepción. También son minoría aquellos estudios que utilizan en sus medidas indicadores derivados del análisis de redes, mostrando sensibilidad por las asimetrías propias del funcionamiento cotidiano de los equipos de trabajo. Una limitación similar se detecta en relación con los estudios multinivel, los cuales aún son relativamente escasos. Parece imprescindible incrementar la investigación sobre cómo las conductas individuales y sus interacciones hacen emerger fenómenos de diferente nivel y cómo éstos influyen sobre las conductas en un nivel colectivo o de equipo (Morgeson y Hofmann, 1999); el ejemplo de la percepción de autoeficacia, la potencia y la eficacia colectiva, así como sus relaciones con el rendimiento individual y de equipo, constituye un caso evidente, pero no por ello aislado en el funcionamiento de los equipos (Lindsley, Brass y Thomas, 1995; Tasa et al., 2007).

En este trabajo hemos partido del modelo IMOI para estructurar nuestra revisión acerca del estado de la investigación sobre la eficacia de los equipos de trabajo, si bien este modelo no está exento de críticas, entre las que destacan su incipiente formulación y la escasez de datos que lo respalden, su radical desviación del tradicional modelo IPO, así como su cuestionamiento de nociones fundamentales en las ciencias sociales tales como la naturaleza de la causación (Salas et al., 2009). No obstante, es un marco conceptual prometedor y de un alto valor heurístico, y teniendo en cuenta la necesidad de integración de la investigación sobre eficacia de los equipos que los principales autores reclaman (Nielsen et al., 2005; Salas et al., 2009; Salas y Wildman, 2009; Weingart y Cronon, 2009), el modelo IMOI puede desempeñar esta función para articular la investigación en la década venidera. 
Tras revisar la literatura científica, nos parece que sería interesante abrir un debate para analizar si los modelos de eficacia de los equipos de trabajo requieren de un mayor carácter contingente que tuviera en cuenta las características y particularidades de cada contexto. Suponer que las variables y procesos de equipo implicados en su eficacia son los mismos en cualquier contexto no parece muy realista, además de contradecir la literatura organizacional, que desde hace décadas ha reconocido y asumido en sus modelizaciones la importancia de los factores contingentes a la hora de diseñar y de analizar la eficacia organizacional. Algún intento de este tipo se está realizando, como ocurre con el desarrollo de un modelo específico de eficacia de equipos en contextos complejos como son los militares (véase, por ejemplo, el modelo CTEF -Command Team Effectiveness- propuesto en el marco de la OTAN por Essens y sus colaboradores, 2009), pero, en general, aún se habla de un modelo de eficacia de equipo sin hacer muchas distinciones relativas a los diferentes contextos.

Un aspecto que requiere de mayor potenciación es el sugerido recientemente por Moreland y Levine (2009) relativo a la necesidad de tender puentes en la investigación sobre grupos, y que puede ampliarse específicamente al caso de los equipos. Así, sería interesante tender puentes a través del tiempo, recuperando modelos y resultados de la investigación tradicional e integrándolos con los actuales; tender puentes entre fenómenos, referido a la necesidad de incluir más variables, tanto de insumos como de procesos y de resultados, en los diseños y no limitarse a analizar relaciones -en ocasiones, obvias- entre unas pocas variables; y tender puentes entre disciplinas, de manera que la riqueza de la investigación sobre equipos realizada en ámbitos diferentes logre una mayor integración, además de evitar que los investigadores de una disciplina "descubran" fenómenos que ya son sobradamente conocidos en otras. Y un último aspecto que los autores señalan: ¿qué debería ser preferible: que los investigadores descubran un nuevo -"su propio"- fenómeno, o que profundicen y desarrollen una mayor comprensión de los fenómenos que ya se han identificado? Aunque puede parecer más excitante, sobre todo para los investigadores más jóvenes, lo primero, quizá sería necesario reflexionar antes de ponerse a descubrir (supuestos) nuevos fenómenos y acuñar novedosos constructos (Moreland y Levine, 2009).

Otra necesidad de integración es la relacionada con la necesidad de una mayor comunicación e interacción entre la investigación académica y la investigación aplicada que permita superar el tradicional "researcher-practitioner gap" existente entre ellas (Anderson y Wheelan, 2005), cuyas principales consecuencias son, por una parte, la intervención ateórica y empirista, sin apoyo conceptual y donde es difícil demostrar las relaciones entre las variables, y por otro, la formulación de modelos teóricos académica y científicamente tan irreprochables como complejos, pero alejados de la realidad de los equipos de las organizaciones y con muy limitadas posibilidades de contrastación empírica.

Por último, un aspecto que también sería necesario potenciar es el relativo al estudio sistemático de las relaciones entre los sistemas de entrenamiento y formación en habilidades y competencias para el trabajo en equipo y sus efectos en la eficacia de los equipos, así como la investigación-intervención enmarcada en las técnicas de consultoría de equipo (Tilin y Sumerson, 2005), dirigidas tanto a la capacitación de los miembros como al desarrollo de equipo.

En conclusión, las demandas reales de las organizaciones actuales y las nuevas formas de organización del trabajo (ej. equipos con alta virtualidad, el papel de la cultura, los nuevos estilos y estrategias para motivar y liderar equipos, los sistemas multi-equipo o el impacto de los equipos sobre la efectividad organizacional) van adelantadas respecto a los desarrollos teóricos y metodológicos. No obstante, el enorme interés que suscitan los tópicos relacionados con los equipos de trabajo como nuevo eje de la gestión organizacional, augura un futuro prometedor para la investigación en este campo.

\section{Extended Summary}

Teams have taken on a central role in organizations over the past four decades. They provide diversity of knowledge, attitudes, skills and experiences, and the integration of these factors allows quick, flexible and innovative responses to problems and challenges, driving performance and enhancing the satisfaction of team members. However, teams do not always operate in this way, and they sometimes fail to achieve the high performance expected of them (Sims, Salas and Burke, 2005).

The main aim of this paper is to synthesize the key results of research into work teams carried out over the last decade from 1999 through 2009. Our approach uses the SWOT technique to identify strengths, weaknesses, opportunities and threats in the field of teamwork research in the next decade. To this end, we set out a brief explanatory framework for the effectiveness of work teams, allowing us to proceed with a differentiated analysis of inputs, mediators and outcomes. Finally, we will integrate and discuss the key challenges facing the field if it is to change threats into opportunities.

\section{Explanatory framework for the effectiveness of work teams}

Numerous reviews of the last decade of research into the effectiveness of work teams have been pub- 
lished recently (e.g. Goodwin, Burke, Wildman and Salas, 2009), all of which, with their differences, are based on the Input-Process-Output (IPO) model. This model identifies membership, structure and processes as the key factors underlying teams' efficacy. At the same time, it establishes that organizational and situational factors influence the structure of the team as a whole, affecting other variables (input, process, output).

\section{Inputs: team resources, task and membership}

Inputs consist of the set of external and internal team resources, which consist of the rewards offered by the organization and organizational culture in the first case, and of team membership (basically members' knowledge and skills), and the structure of the group and design of the task in the second. These resources can also be considered on different levels (the resources of team members, the group and the organization).The following SWOT analysis summarizes findings in the area of inputs (see Table 1).

\section{Mediators: processes, emerging states and mixed mediators}

Mediators consist of a series of psychosocial mechanisms that allow the members of a team to combine the available resources to carry out the task assigned by the organization, overcoming the difficulties inherent in coordination and motivation of each individual. Research has accumulated in this area, and despite the current distinction between processes, emerging states and mixed mediators (Mathieu et al., 2008), the literature has not yet reached a consensus classification of mediators. The following SWOT analysis summarizes findings in the area of mediators (see Table 2).

\section{Team outcomes}

Team outcomes are usually viewed from a multidimensional and multi-level standpoint (Mathieu et al., 2008).In general, efficacy is analyzed in terms of work outcomes, such as objectively assessed performance. However, the analysis may also include outcomes that help keep a team functioning well over time, such as

Table 1. SWOT analysis for inputs

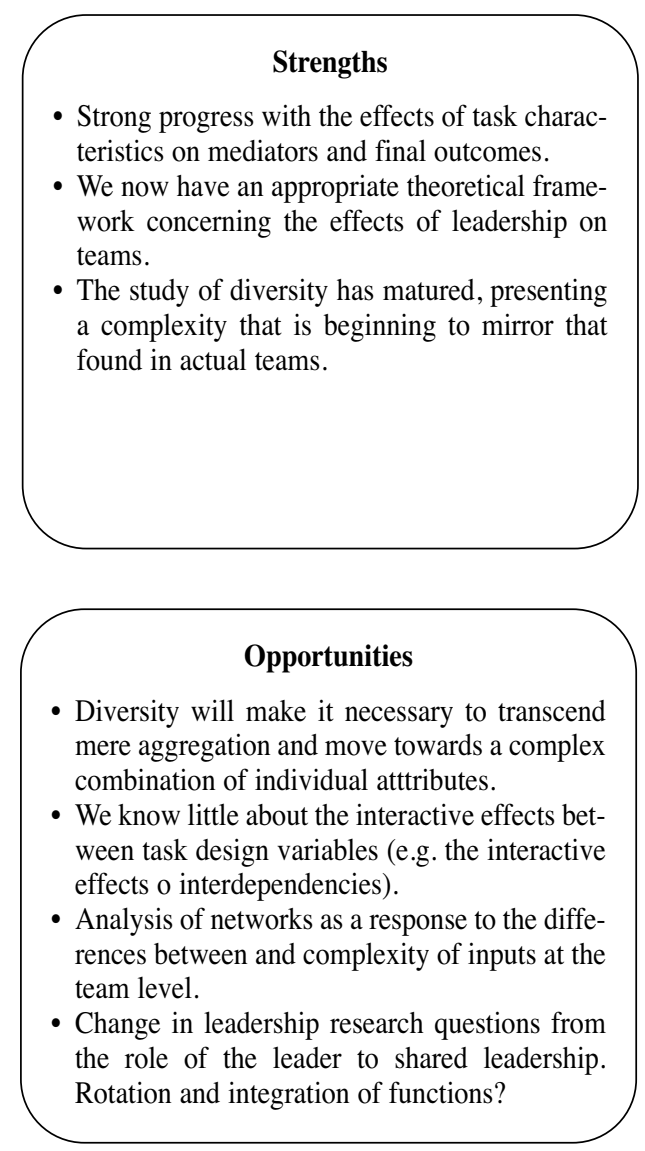

\section{Weaknesses}

- The samples needed to study inputs must be sufficiently varied. It is not the quantity but the quality of the data that matters.

- While we assume a unitary definition of inputs, we tend to pass over asymmetries and important differences (e.g. diversity, interdependence and virtuality).

- We assume equal contributions from the different members of the team.

- Studies of virtuality assume the direct transfer of theories developed in the area of traditional teams.

\section{Threats}

- The role of time on the effect of inputs has scarcely been examined to date.

- Changes in the composition of teams and multiple team membership are common in organizations, but not on the research agenda.

- Multinational corporations create multinational teams, which are diferentially affected by diversity and culture.

- The dynamics of today's organizations threaten our static approach to research. 


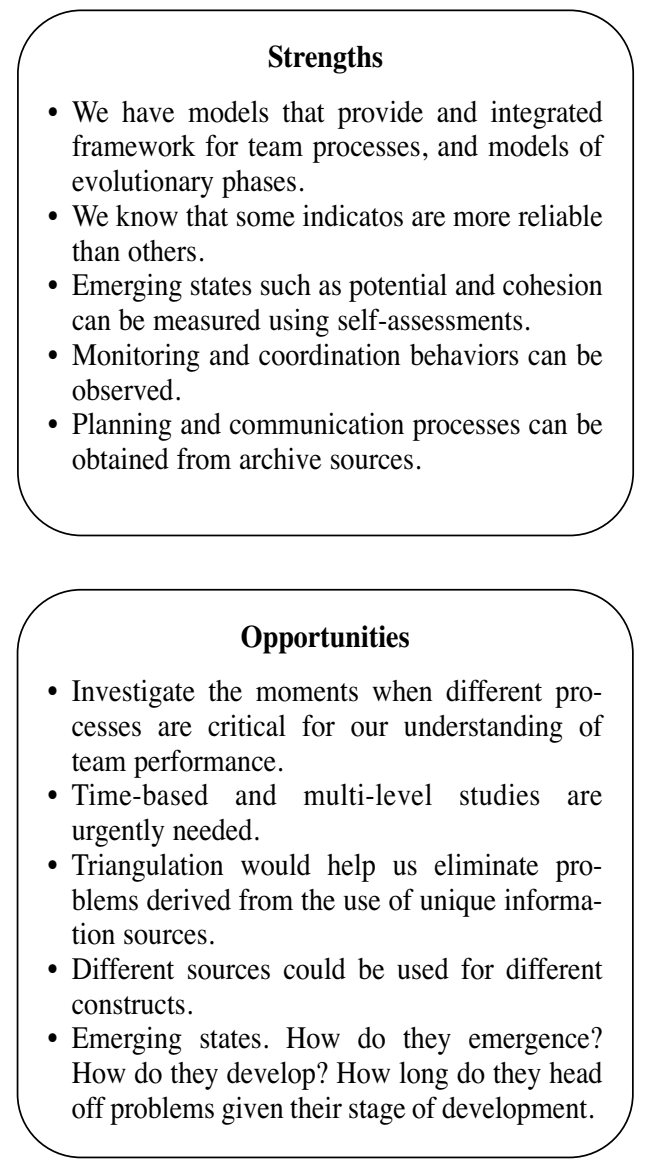

member satisfaction, viability (the extent to which team members wish to stay together) and innovation (Gil, Rico and Sánchez-Manzanares, 2008). Meanwhile, the literature has systematically sought to identify antecedent and mediating variables, while largely ignoring the classification of outcomes (Mathieu et al., 2008).In this light, we take an approach that summarizes team results at the level of the organization, the team and the role, and for three basic types of outcome, consisting of functioning (relevant actions to achieve results), performance (the consequences of functioning) and attitudes. We propose a $3 \times 3$ matrix to reflect the evolution of the literature in this area. The following SWOT analysis summarizes findings in the area of outcomes (see Table 3).

\section{Discussion}

Current theoretical approaches agree in treating teams as complex adaptive systems, the efficacy of which depends on interactions between different inputs, processes, derived emerging states, mixed mediators and outcomes. However, studies in the field

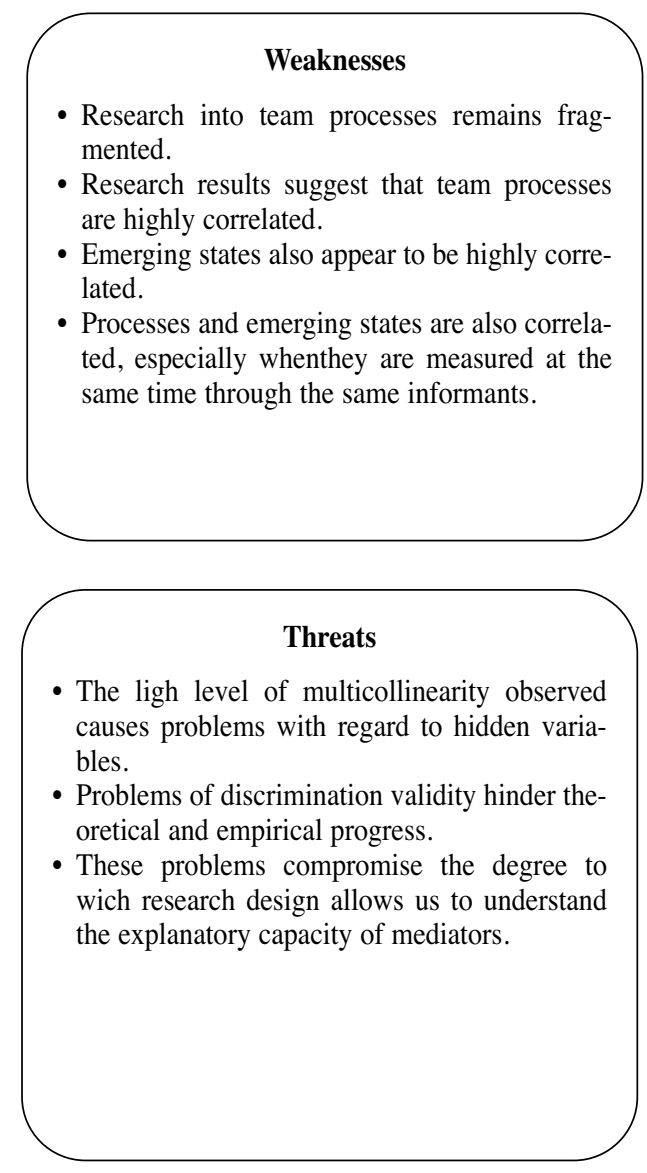

of work teams have tended to concentrate on inputs and outcomes, although the research effort and progress have been more limited in the latter case. Although research into mediators has been proportionally less intense, processes and emerging states have received increasing attention from researchers in recent years. This has resulted in decisive progress in the study of team cognition, although more work is needed to identify the antecedents and effects of cognitive processes, to establish evaluation methods and to improve processes of this kind. Theoretical developments concerning emerging emotional states remain precarious, and few studies have examined their impact on team efficacy. Finally, longitudinal studies focusing on dynamic issues of team belonging continue to be the exception, despite the importance attached to the long-run functioning of teams. Meanwhile, only a minority of studies use measures and indicators derived from network analysis, reflecting an awareness of the asymmetries inherent in the day-to-day functioning of work teams. A similar limitation may be observed in relation to multi-level studies, which are very thin on the ground. It would seem essential, then, to foster research into the ways in which individual behaviors and their interactions result in the emergence 


\section{Strengths}

- Appropriate differentiation of theam-level and role-demand based outcomes, allowing comparison between teams.

- Developtment of multi-level models allowing the creation of effectiveness measures at the level ot the team.
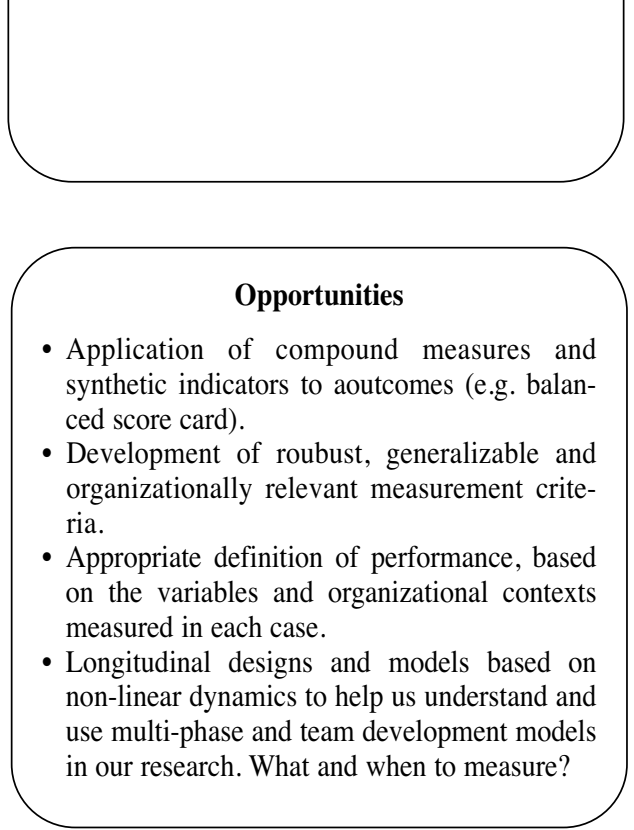

of phenomena on different levels, and how these influence behaviors at the collective or group level (Morgeson and Hofmann, 1999). The perception of self-efficacy, collective potential and efficacy, and the relationship of this factor with individual performance is a case in point, but this does not mean that it is isolated from overall team performance (Lindsley, Brass and Thomas, 1995; Tasa et al., 2007).

\section{Referencias}

Allen, B. C., Sargent, L. D. y Bradley, L. M. (2003). Differential effects of task and reward interdependence on perceived helping behavior, effort, and group performance. Small Group Research, 34, 716-740.

Ancona, D. G. y Caldwell, D. F. (1992). Demography and design: Predictors of new product team performance. Organization Science, 3, 321-341.

Anderson, G. y Wheelan, S. A. (2005). Integrating group research and practice. En S. A. Wheelan (Ed.), The Handbook of Group Research and Practice (pp.545-552). Thousand Oaks, CA: Sage.

Anderson, N. y West, M. (1998).Measuring climate for work group innovation: development and validation of the

\section{Weaknesses}

- Use of poorly specified self-reporting criteria.

- Scant attention to the measurement of affective outcomes over the past decade.

- Discrimination validity problems, especally between measures of affective outcomes.

- We not clearly understand the dynamics of time in team outcomes.

\section{Threats}

- Scant evidence for the impact of outcomes at the organizational level. Wht is the actual contribution made by teams?

- Organizations are increasingly interested in understanding the effective coordination of multi-team systems with a view to attaining organizational goals.

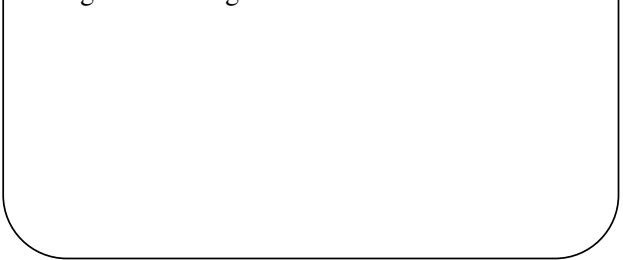

team climate inventory. Journal of Organizational Behavior, 19, 235-58.

Aritzeta, A. y Alcover, C. M. (2006). ¿Quién decide? ¿Quién es responsable? ¿Quién gana? Análisis de las dificultades para la implementación de equipos de trabajo. Revista de Psicología del Trabajo y de las Organizaciones, 22, 151-178.

Arrow, H., McGrath, J. E. y Berdahl, J. L. (2000). Small groups as complex systems. Thousand Oaks, CA: Sage.

Aubert, B. y Kelsey, B. (2003). Further understanding of trust and performance in virtual teams. Small Group Research, 34, 575-619.

Austin, J. R. (2003). Transactive memory in organizational groups: The effects of content, consensus, specialization and accuracy on group performance. Journal of Applied Psychology, 88, 866-878.

Bachrach, D. G., Powell, B. C., Collins, B. J. y Richey, R. G.(2006). Effects of task interdependence on the relationship between helping behavior and group performance. Journal of Applied Psychology, 91, 1396-1405.

Balkundi, P. y Harrison, D. A. (2006). Ties, leaders, and time in teams: Strong inference about network structure's effects on team viability and performance. Academy of Management Journal, 49, 49-68.

Barrick, M. B., Bradley, B. H., Kristof-Brown, A. L. y 
Colbert, A. E. (2007). The moderating role of top management team interdependence: Implications for real teams and working groups. Academy of Management Journal, 50, 544-557.

Bass, B. M., Avolio, B. J., Jung, D. I. y Berson, Y. (2003). Predicting unit performance by assessing transformational and transactional leadership. Journal of Applied Psychology, 88, 207-218.

Beal, D. J., Cohen, R. R., Burke, M. J. y McLendon, C. L. (2003). Cohesion and performance in groups: A metaanalytic clarification of construct relations. Journal of Applied Psychology, 88, 989-1004.

Bolino, M. C., Turnley, W. H. y Bloodgood, J. M.(2002). Citizenship behavior and the creation of social capital in organizations. Academy of Management Review, 27, 50522.

Brannick, M. T. y Prince, T. (1997). An overview of team performance measurement. En E. Salas y C .Prince (Eds.), Team performance assessment and measurement(pp.3-16). Mahwah, NJ: Lawrence Erlbaum Associates.

Brannick, M. T., Prince, A., Prince, C. y Salas, E. (1995). The measurement of team process. Human Factors,37, 641- 651.

Bunderson, J. S. y Sutcliffe, K. A. (2002).Comparing alternate conceptualizations of functional diversity in management teams: Process and performance. Academy of Management Journal, 45, 875-893.

Burningham, C. y West, M. (1995). Individual, climate, and group interaction processes as predictors of work team innovation. Small Group Research, 26, 106-117.

Burke, C. S., Stagl, K. C., Klein, C., Goodwin, G. F., Salas, E. y Halpin, S.M. (2006). What type of leadership behaviors are functional in teams? A meta-analysis. Leadership Quarterly, 17, 288-307.

Campion, M. A., Papper, E. M. y Medsker, G. J. (1996). Relations between work team characteristics and effectiveness: A replication and extension. Personnel Psychology, 49, 429-452.

Cannon-Bowers, J. A., Salas, E. y Converse, S. A. (1993). Shared mental models in expert decision-making teams. En N.J. Castellan (Ed.), Current issues in individual and group decision making (pp.221-246). Hillsdale, NJ: Lawrence Erlbaum Assoc.

Cannon-Bowers, J. A., Tannenbaum, S. I., Salas, E. y Volpe, C.E. (1995). Defining competencies and establishing team training requirements. En R.A. Guzzo y E. Salas (Eds.), Team Effectiveness and Decision Making in Organizations (pp. 333-380). San Francisco: Jossey-Bass.

Carson, J. B., Tesluk, P. E. y Marrone, J. A. (2007). Shared leadership in teams: An investigation of antecedent conditions and performance. Academy of Management Journal, 50, 1217-1234.

Chen, G. (2005). Newcomer adaptation in teams: Multilevel antecedents and outcomes. Academy of Management Journal, 48, 101-116.

Chen, G., Kirkman, B.L., Kanfer, R., Allen, D. y Rosen, B. (2007). A multilevel study of leadership, empowerment, and performance in teams. Journal of Applied Psychology, 92, 331-346.

Chiochio, F. y Essiembre, H. (2009). Cohesion and performance: A meta-analytic review of disparities between project teams, production teams, and service teams. Small Group Research, 40, 382-420.

Cohen, S. G. y Bailey, D. E. (1997.) What makes teams work: Group effectiveness research from the shop floor to the executive suite. Journal of Management, 23, 239-290.

Colquitt, J. A., Conlon, D.E., Wesson, M.J., Porter, C. y Ng, K.Y. (2001). Justice at the Millennium: A meta-analytic review of 25 years of organizational justice research. Journal of Applied Psychology, 86, 425-445.

Colquitt, J. A., Noe, R. A. y Jackson, C. L. (2002). Justice in teams: Antecedents and consequences of procedural justice climate. Personnel Psychology, 55, 83-109.

Cooke, N. J., Gorman, J. C. y Winner, J. L. (2007).Team Cognition. En F.T. Durso. Handbook of Applied Cognition, $2^{\text {nd }} E d$. (pp. 239-268). NY: John Willey \& Sons.

Coppola, N. W., Hiltz, S. R. y Rotter, N. G. (2004) Building trust in virtual teams. IEEE Transactions on Professional Communication, 47, 95-104.

Day, D. V., Gronn, P. y Salas, E. (2004). Leadership capacity in teams. The Leadership Quarterly, 15,857-880.

Day, E. A., Arthur, W. J., Miyashiro, B., Edwards, B. D., Tubre, T. C. y Tubre, A. H. (2004). Criterion-related validity of statistical operationalizations of group general cognitive ability as a function of task type: Comparing the mean, maximum, and minimum. Journal of Applied Social Psychology, 34, 1521-1549.

De Dreu, C. K. W. (2007). Cooperative outcome interdependence, task reflexivity, and team effectiveness: A motivated information processing perspective. Journal of Applied Psychology, 92, 628-638.

De Dreu, C. K. W. y Weingart, L. R. (2003). Task versus relationship conflict: Team performance, and team member satisfaction: A meta-analysis. Journal of Applied Psychology, 88, 741-749.

De Dreu, C. K. W. y West, M. A. (2001). Minority dissent and team innovation: The importance of participation in decision making. Journal of Applied Psychology, 86, 1191-1201.

De Jong, S. B., van der Vegt, G. S. y Molleman, E. (2007). The relationships among asymmetry in task dependence, perceived helping behavior, and trust. Journal of Applied Psychology, 92, 1625-37.

De Rosa, D. M., Hantula, D. A., Dock, N. y D'Arcy, J. (2004). Trust and leadership in virtual teamwork: a media naturalness perspective. Human Resource Management, 43, 219-32.

DeChurch, L. A. y Mesmer-Magnus, J. R. (En prensa). The Cognitive Underpinnings of Effective Teamwork: A Meta-Analysis. Journal of Applied Psychology.

DeRue, D. S., Hollenbeck, J.R., Johnson, M. D., Ilgen, D. R. yJundt, D. K. (2008). How different team downsizing approaches influence team-level adaptation and performance. Academy of Management Journal, 51, 182-96. 
Dirks, K. T. y Ferrin, D. L. (2001).The role of trust in organizational settings. Organizational Science, 12, 450-67.

Druskat, V. U. y Kayes, D. C. (2000).Learning versus performance in short-term project teams. Small Group Research, 31, 328-353.

Eckel, C. C. y Grossman, P. J. (2004).Managing diversity by creating team identity. Journal of Economic Behavior and Organization, 58, 317-392.

Edmondson, A. C. (1999). Psychological safety and learning behaviors in work teams. Administrative Science Quarterly, 44, 350-383.

Edwards, B. D., Day, E. A., Arthur, W. y Bell, S. T. (2006). Relationships among team ability composition, team mental models, and team performance. Journal of Applied Psychology, 91, 727-736.

Ellis, A. P. J. (2006.) System breakdown: The role of mental models and transactive memory in the relationship between acute stress and team performance. Academy of Management Journal, 49, 576-589.

Ensley, M. D., Hmieleski, K. M. y Pearce, C. L. (2006). The importance of vertical and shared leadership within new venture top management teams: Implications for the performance of startups. Leadership Quarterly, 17, 217-231.

Espinosa, J. A., Lerch, J. y Kraut, R. (2004). Explicit vs. implicit coordination mechanisms and task dependencies: One size does not fit all. En E. Salas y S.M. Fiore (Eds.), Team Cognition: Understanding the Factors that Drive Process and Performance (pp.107-129). Washington, DC: APA Books.

Essens, P. J. M. D., Vogelaar, Ad. L. W., Mylle, J. J. C., Blendell, C., Paris, C., Halpin, S. M. y Baranski, J. V. (2009). Team effectiveness in complex settings: A framework. En E. Salas, G. F. Goodwin y C. S. Burke (Eds.), Team Effectiveness in Complex Organizations. CrossDisciplinary Perspectives and Approaches (pp. 293-320). Nueva York: Psychology Press.

Gibson, C. B. y Vermeulen, F. (2003). A healthy divide: Subgroups as stimulus for team learning behavior. Administrative Science Quarterly, 48, 202-239.

Gibson, C. B. (2003). Quality of team service-The role of field independent culture, quality orientation, and quality improvement focus. Small Group Research, 34, 619-646.

Gibson, C. B. y Manuel, J. A. (2003). Building trust: effective multicultural communication processes in virtual teams. En C. B. Gibson y S. G. Cohen (Eds.) Virtual teams that work: creating conditions for virtual teams effectiveness (pp. 59-86. San Francisco, CA: Jossey-Bass.

Gibson, C. B., Porath C.L., Benson, G. S. y Lawler, E. E. (2007). What results when firms implement practices: The differential relationship between specific practices, firm financial performance, customer service, and quality. Journal of Applied Psychology, 92, 1467-1480.

Gil, F., Alcover, C. M ${ }^{\mathrm{a}}$.y Peiró, J. M. (2005). Work team effectiveness in organizational contexts: Recent research and applications in Spain and Portugal. Journal of Managerial Psychology, 20, 193-218.

Gil, F., Rico, R. y Sánchez-Manzanares, M. (2008). Eficacia de equipos de trabajo. Papeles del Psicólogo, 29, 25-31.
Gilson, L. L. y Shalley, C. E. (2004). A little creativity goes a long way: An examination of teams' engagement in creative processes. Journal of Management, 30, 453-470.

Golembiewski, R. T. y McConkie, M. (1975). The centrality of interpersonal trust in group processes. En C.L. Cooper (Ed.) Theories of group processes (pp. 131-85). New York: Wiley.

González-Romá, V., Peiró, J. M. y Tordera, N. (2002). An examination of the antecedents and moderator influences of climate strength. Journal of applied psychology, 87, 465-73.

Goodwin, G. F., Burke, C. S., Wildman, J. L. y Salas, E. (2009). Team effectiveness in complex organizations: An overview. En E. Salas, G.F. Goodwin y C.S. Burke (Eds.), Team Effectiveness in Complex Organizations. CrossDisciplinary Perspectives and Approaches (pp. 3-16). New York: Psychology Press.

Gully, S. M., Incalcaterra, K. A., Joshi, A. y Beaubien, J. M. (2002). A meta-analysis of team-efficacy, potency and performance: Interdependence and level of analysis as moderators of observed relationships. Journal of Applied Psychology, 87, 819-832.

Guzzo, R. A., y Dickson, M. W. (1996). Teams in organization: Recent research on performance and effectiveness. Annual Review of Psychology, 47, 307-338.

Hackman, J. R. y Wageman, R. (2005). A theory of team coaching. Academy of Management Review, 30, 269-287.

Hackman, J. R. (1983). A normative model of work team effectiveness (Technical Report No. 2). New Haven, CT: Yale School of Organization and Management.

Hackman, J. R. (2002). Leading teams: Setting the stage for great performances. Boston: Harvard Business School Press.

Hackman, J. R. (1998). Why teams don't work. En S. R. Tindale, L. Heath, J. Edwards, E. J. Posavac, F. B. Bryant, Y. Suarez-Balcazar, E. Henderson-King y J. Myers (Eds.), Theory and Research on Small Groups (pp. 245-266). New York: Plenum Press.

Harrison, D., Price, K. H., Gavin, J. H. y Florey, A. T. (2002). Time, teams, and task performance: changing effects of surface and deep-level diversity on group functioning. Academy of Management Journal, 45, 1029-45.

Hiller, N. J., Day, D. V. y Vance, R. J. (2006). Collective enactment of leadership roles and team effectiveness: A field study. Leadership Quarterly, 17, 387-397.

Hinds, P. J. y Bailey, D. E. (2003).Out of sight, out of sync: understanding conflict on distributed teams. Organization science, $14,615-32$.

Høigaard, R., Säfvenbom, R. y Tønnessen, F. E. (2006). The relationship between group cohesion, group norms, and perceived social loafing in soccer teams. Small Group Research, 37, 217-232.

Hollenbeck, J. R. (2009). Dealing with common pitfalls and misconceptions in team research. Paper presented at the $69^{\text {th }}$ Academy of Management Meeting. Chicago, Il: EE.UU.

Homan, A., van Knippenberg, D., van Kleef, G. A. y De Dreu, C. K. W. (2007). Bridging faultlinesby valuing 
diversity: Diversity beliefs, information elaboration, and performance in diverse work groups. Journal of Applied Psychology, 92, 1189-1199.

House, R., Javidan, M., Hanges, P. y Dorfman, P. (2002). Understanding cultures and implicit leadership theories across the globe: An introduction to project GLOBE. Journal of World Business, 37, 3-10.

Hülsheger, U. R., Anderson, N. y Salgado, J. F. (2009). Team-level predictors of innovation at work: a comprehensive meta-analysis spanning three decades of research. Journal of Applied Psychology, 94, 1128-1145.

Hyatt, D. E.y Ruddy, T. M. (1997). An examination of the relationship between work group characteristics and performance: Once more into the breech. Personnel Psychology, 50, 553-585.

Ilgen, D. R., Hollenbeck, J. R., Johnson, M. yJundt, D. (2005). Teams in organizations: From I-P-O models to IMOI models. Annual Review of Psychology, 56, 517544.

Jackson, S. E. (1996). The consequences of diversity in multidisciplinary work teams. En M. A. West (Ed.), The psychology of groups at work (pp. 53-76). Chichester, UK: John Wiley \& Sons.

Jackson, S. E., Joshi, A. y Erhardt, N. L. (2003). Recent research on teams and organizational diversity: SWOT analysis and implications. Journal of Management, 29, 801-830.

Jackson, S. E., y Joshi, A. (2004). Diversity in social context: A multi-attribute, multi-level analysis of team diversity and performance. Journal of Organizational Behavior, 25, 675-702.

Janicik, G. A. y Bartel, C. A. (2003). Talking about time: Effects of temporal planning and time awareness norms on group coordination and performance. Group Dynamics: Theory, Research, and Practice, 7, 122-134.

Jarvenpaa, S. L. y Leidner, D. E. (1999). Communication and trust in global virtual teams. Organizational science, $10,791-815$.

Jarvenpaa, S. L., Shaw, T. T. y Staples, D. S. (2004). Toward contextualized theories of trust: The role of trust in global virtual teams. Information System Research, 15, 25067.

Jehn, K. A. y Bezrukova, K. (2004). A field study of group diversity, group context, and performance. Journal of Organizational Behavior, 25, 1-27.

Jehn, K. A. (1995). A multimethod examination of the benefits and detriments of intragroup conflict. Administrative Science Quarterly, 40, 256-282.

Jehn, K. A. y Shah, P. P. (1997).Interpersonal relationships and task performance. An examination of mediation processes in friendship and acquaintance groups. Journal of Personality and Social Psychology, 72, 775-790.

Jehn, K. A., Northcraft, G. B. y Neale, M. A. (1999). Why differences make a difference: A field study of diversity, conflict, and performance in workgroups. Administrative Science Quarterly, 44, 741-763.

Johnson, M. D., Hollenbeck, J. R., Humphrey, S. E., Ilgen, D. R., Jundt, D. y Meyer, C. J. (2006). Cutthroat cooper- ation: Asymmetrical adaptation to changes in team reward structures. Academy of Management Journal, 49, 103-119.

Katz, N., David L., Holly A. y Contractor, N. (2004). The network perspective on teams. Small Group Research, 35, 307-332.

Kennedy, F. A., Loughry, M. L., Klammer, T. P. y Beyerlein, M. M. (2009). Effects of organizational support on potency in work teams: The mediating role of team processes. Small Group Research, 40, 72-93.

Kirkman, B. L. y Rosen, B. (1999). Beyond self-management: Antecedents and consequences of team empowerment. Academy of Management Journal, 42, 58-74.

Kirkman, B. L., Rosen, B., Gibson, C. B., Tesluk, P. E. y McPherson, S. O. (2002). Five challenges to virtual team success: lessons from Sabre, Inc.'. Academy of Management Executive, 16, 67-79.

Kirkman, B. L., Rosen, B., Tesluk, P. E. y Gibson, C. B. (2004). The impact of team empowerment on virtual team performance: The moderating role of face-to-face interaction. Academy of Management Journal, 47, 175-192.

Kirkman, B. L., Tesluk, P. E. y Rosen, B. (2004). The impact of demographic heterogeneity and team leader-team member demographic fit on team empowerment and effectiveness. Group and Organization Management, 29, 334-368.

Kirkman, B. L., y Mathieu, J. E. (2005). The dimensions and antecedents of team virtuality. Journal of Management, 31, 700-718.

Kochan, T., Bezrukova, K., Ely, R., Jackson, S., Joshi, A., Jehn, K., Leonard, J., Levine, D. y Thomas, D. (2003). The effect of diversity on business performance: Report of the diversity research network. Human Resources Management, 42, 3-21.

Kozlowski, S. W. J., Gully, S. M., Nason, E. R. y Smith, E. M. (1999). Developing adaptative teams: A theory of compilation and performance across levels and time. En D. R. Ilgen y E. D. Pulatos (Eds.), The Changing Nature of Work and Performance: Implications for Staffing, Personnel Actions, and Development. San Francisco: Jossey-Bass.

Kozlowsi, S. W. J e Ilgen, D. R. (2006). Enhancing the effectiveness of work groups and teams. Psychological Science in the Public Interest, 7, 77-124.

Kozlowski, S. W. J. y Bell, B. (2003). Work groups and teams in organizations. En W. C. Borman, D. R. Ilgen y R. J. Klimoski (Eds.), Industrial and Organizational Psychology (pp. 333-375).Chichester: John Wiley \&Sons.

Kozlowski, S. W. J. y Klein, K. J. (2000).A multilevel approach to theory and research in organizations: Contextual, temporal, and emergent processes. En K.J. Klein y S. W. J. Kozlowski (Eds.), Multilevel theory, research, and methods in organizations (pp. 3-90). San Fran-cisco: Jossey-Bass.

Langfred, C. W. (2000). Work-group design and autonomy - A field study of the interaction between task interdependence and group autonomy. Small Group Research, $31,54-70$. 
Langfred, C. W. (2005). Autonomy and performance in teams: The multilevel moderating effect of task interdependence. Journal of Management, 31, 513-529.

Lau, D. y Murnighan, J. K. (1998). Demographic diversity and faultlines: The compositional dynamics of organizational groups. Academy of Management Review, 23, 325340.

Lau, D. y Murnighan, J. K. (2005). Interactions within groups and subgroups: The effects of demographic faultlines. Academy of Management Journal, 48, 645-659.

Lawler, E. E. y Worley, Ch. G. (2006). Built to change. How to achieve sustained organizational effectiveness. San Francisco: Jossey-Bass

LePine, J. A. (2003). Team adaptation and post-change performance: Effects of team composition in terms of members' cognitive ability and personality. Journal of Applied Psychology, 88, 27-39.

LePine, J. A., Piccolo, R. F., Jackson, C. L., Mathieu, J. E. y Saul, J.R. (2008). A meta-analysis of teamwork processes: Tests of a multidimensional model and relationships with team effectiveness criteria. Personnel Psychology, 61, 273-307.

Levine, J. M. y Moreland, R. L. (1990). Progress in small group research. Annual Review of Psychology, 41, 585634.

Lewis, K. (2004). Knowledge and performance in knowledge-worker teams: A longitudinal study of transactive memory systems. Management Science, 50, 1519-1533.

Lewis, K., Belliveau, M., Herndon, B. y Keller, J. (2007). Group cognition, membership change, and performance: Investigating the benefits and detriments of collective knowledge. Organizational Behavior and Human Decision Processes, 103, 159-178

Lewis, K., Lange, D. y Gillis, L. (2005). Transactive memory systems, learning, and learning transfer. Organization Science, 16, 581-598.

Li, J. T. y Hambrick, D. C. (2005). Factional groups: A new vantage on demographic faultlines, conflict, and disintegration in work teams. Academy of Management Journal, 48, 794-813.

Lim, B. C. y Klein, K. J. (2006). Team mental models and team performance: A field study of the effects of team mental model similarity and accuracy. Journal of Organizational Behavior, 27, 403-418.

Lindsley, D. H., Brass, D. J. y Thomas, J. B. (1995). Efficacy-performance spirals: A multilevel perspective. Academy of Management Review, 20, 645-678.

Lira, E. M., Ripoll, P., Peiró, J. M. y González, P. (2007). The roles of group potency and information and communication technologies in the relationship between task conflict and team effectiveness: A longitudinal study. Computers in Human Behavior, 23, 2888-2903.

Malone, T. W. y Crowston, K. (1994).The interdisciplinary study of coordination. ACM Computing Surveys,26, 87119.

Marks, M.A ., Mathieu, J. E., y Zaccaro, S. J. (2001). A temporally based framework and taxonomy of team processes. Academy of Management Review, 26, 356-376.
Marks, M. A., Mathieu, J. E., Alonso, A., DeChurch, L. y Panzer, F. J. (2005). Teamwork in multiteam systems. Journal of Applied Psychology, 90, 964-971.

Martins, L. L., Gilson, L .L. y Maynard, M. T. (2004) Virtual teams: what do we know and where do we go from here? Journal of management, 6, 805-35.

Martins, L. L., Gilson, L. L., y Maynard, M. T. (2004). Virtual teams: What do we know and where do we go from here? Journal of Management, 30, 805-835.

Maruping, L. M. y Agarwal, R.(2004). Managing team interpersonal processes through technology: A task-technology fit perspective. Journal of Applied Psychology, 89, 975-90.

Mathieu, J., Maynard, M. T., Rapp, T. y Gilson, L. (2008). Team effectiveness 1997-2007: A review of recent advancements and a glimpse into the future. Journal of Management, 34, 410-476.

Mathieu, J. E. y Rapp, T. L. (2009). Laying the foundation for successful team performance trajectories: The role of team charters and deliberate plans. Journal of Applied Psychology, 94,90-103.

Mathieu, J. E. y Schulze, W. (2006). The influence of team knowledge and formal plans on episodic team processàperformance relationships. Academy of Management Journal, 49, 605-619.

Mathieu, J. E., Heffner, T. S., Goodwin, G. F., Cannon-Bowers, J.A. y Salas, E. (2005).Scaling the quality of teammates'mental models: Equifinality and normative comparisons. Journal of Organizational Behavior, 26, 37-56.

Mathieu, J. E., Maynard, M. T., Taylor, S. R., Gilson, L. L. y Ruddy, T. M. (2007). An examination of the effects of organizational district and team contexts on team processes and performance: A meso-mediational model. Journal of Organizational Behavior, 28, 891-910.

Mayer, R. C., Davis, J. H. y Schoorman, F. D. (1995). An integrative model of organizational trust. Academy of Management Review, 20, 709-734.

McGrath, J. E., Arrow, H. y Berdahl, J. L. (2000). The study of groups: Past, present, and future. Personality and Social Psychology Review, 4, 95-105.

McGrath, J. E. (1964). Social psychology: A brief introduction. New York: Holt, Rinehart \& Winston.

McGrath, J. E. y Argote, L. (2001). Group processes in organizational contexts. En M.A. Hogg y R.S. Tindale (Eds.), Blackwell handbook of social psychology: Group processes, vol. 3 (pp. 603-627). Oxford: Blackwell.

McIntyre, R. M. y Salas, E. (1995). Measuring and managing for team performance: Emerging principles from complex environments. En R.A. Guzzoy E. Salas (Eds.), Team effectiveness and decision making in organizations. San Francisco: Jossey-Bass.

McKnight, D. H. y Chervany, N. L. (2006).Reflections on an initial trust-building model. En R. Bachman y A. Zaheer (Eds.) Handbook of Trust Research, (pp. 29-51). Cheltenham: Edward Elgar.

McKnight, D. H., Cummings, L. L. y Chervany, N. L. (1998). Initial trust formation in new organizational relationships. Academy of Management Review, 23, 1-18. 
Mehra, A., Smith, B., Dixon, A. y Robertson, B. (2006). Distributed leadership in teams: The network of leadership perceptions and team performance. Leadership Quarterly, 17, 232-245.

Michel, J. y Hambrick, D. (1992).Diversification posture and the characteristics of the top management team. Academy of Management Journal, 35, 9-37.

Milliken, F. y Martins, L. (1996). Searching for common threads: Understanding the multiple effects of diversity in organizational groups. Academy of Management Review, 21, 402-433.

Mohrman, S. A., Cohen, S. G. y Mohrman, A. M. (1995). Designing Team-based Organizations: New Forms for Knowledge Work. San Francisco: Jossey-Bass.

Moreland, R. L. y Levine, J. M. (2009).Buiding bridges to improve theory and research on small groups. En E. Salas, G. F. Goodwin y C. S. Burke (Eds.), Team Effectiveness in Complex Organizations. CrossDisciplinary Perspectives and Approaches (pp. 17-38). Nueva York: Psychology Press.

Morgeson, F. P. (2005). The external leadership of self-managing teams: Intervening in the context of novel and disruptive events. Journal of Applied Psychology, 90, $497-$ 508 .

Morgeson, F. P. y Hofmann, D. A. (1999). The structure and function of collective constructs: Implications for multilevel research and theory development. Academy of Management Review, 24, 249-265

Neal, A. y Griffin, M. A. (2006). A study of the lagged relationships among safety climate, safety motivation, safety behavior, and accidents at the individual and group levels. Journal of Applied Psychology, 91, 946-953.

Newell, S., David, G. y Chand, D. (2007). An analysis of trust among globally distributed work teams in an organizational setting. Knowledge and Process Management, $14,158-68$.

Nielsen, T. M., Sundstrom, E. D. y Halfhill, T. R. (2005). Group dynamics and effectiveness: Five years of applied research. En S. A. Wheelan (Ed.), The Handbook of Group Research and Practice (pp.285-311). Thousand Oaks, CA: Sage.

O'Reilly, C. A., Caldwell, D. y Barnett, W. (1989). Work group demography, social integration, and turnover. Administrative Science Quarterly, 34, 21-37.

Organ, D. W. (1997). Organizational citizenship behavior: Its construct clean-up time. Human Performance, 10, 8597.

Organ, D. W., Podsakoff, P.M. y MacKenzie, S. B. (2006). Organizational Citizenship Behavior: Its Nature, Antecedents and Consequences. Beverly Hills, CA: Sage

Pearce, J. L. y Gregersen, H. B. (1991). Task interdependence and extra-role behavior: A test of the mediating effects of felt responsibility. Journal of Applied Psychology, 76, 838-844.

Perretti, F. y Negro, G. (2007). Mixing genres and matching people: A study of innovation and team composition in Hollywood. Journal of Organizational Behavior, 28, 563586.
Pirola-Merlo, A., Hartel, C., Mann, L. y Hirst, G. (2002). How leaders influence the impact of affective events on team climate and performance in $\mathrm{R} \& \mathrm{D}$ teams. The Leadership Quarterly, 13, 561-581.

Porter, C. O. L. H. (2005). Goal orientation: Effects on backing up behavior, performance, efficacy, and commitment in teams. Journal of Applied Psychology, 90, 811-818.

Pritchard, R. D. (1995). Lessons learned about ProMES. En R. D. Pritchard (Ed.), Productivity Measurement and Improvement: Organizational Case Studies (pp.325-365). New York: Praeger.

Pritchard, R. D., Jones, S., Roth, P., Stuebing, K. y Ekeberg, S. (2001). Effects of group feedback, goal setting, and incentives on organizational productivity. Journal of Applied Psychology, 73, 337-358.

Probst, G. J. B. y Büchel, B. S. T. (1997). Organizational Learning. Londres: Prentice Hall.

Rico, R., Alcover, C. M., Sánchez-Manzanares, M. y Gil, F. (2009). The joint relationships of communication behaviors and task interdependence on trust building and change in virtual project teams. Social Science Information, 48, 229-255.

Rico, R. y Cohen, S. G.(2005). Effects of task interdependence and type of communication on performance in virtual teams. Journal of Managerial Psychology, 20 (3/4), 261-274.

Rico, R., Cohen, S. G. y Gil, F. (2006). Efectos de la interdependencia de tarea y la sincronía en las tecnologías de comunicación sobre el rendimiento de los equipos virtuales de trabajo. Psicothema, 18, 743-749.

Rico, R. y DeChurch, L. A. (Enviado). Moving beyond teams: a multilevel model of multiteam performance. Paper to be presented at SIOP Annual Conference, Atlanta: EE.UU. (2010).

Rico, R., Molleman, E., Sánchez-Manzanares, M. y van der Vegt, G. (2007). The effects of diversity faultlines and team task autonomy on decision quality and social integration. Journal of Management, 33, 111-132.

Rico, R., Sánchez-Manzanares, M., Gil, F. y Gibson, C. (2008). Team implicit coordination processes: A team knowledge based approach. Academy of Management Review, 33, 163-184.

Rothrock, L., Cohen, A., Yin, J., Thiruvengada, H. y NahumShani, I. (2009). Analyses of team performance in a dynamic task environment. Applied Ergonomics, 40, 699706.

Rousseau, D. M., Sitkin, S. B., Burt, R. S. y Camerer, C. (1998). Not so different after all: a cross-discipline view of trust. Academy of Management Review, 23, 393-404.

Saavedra, R., Earley, P.C. y Van Dyne, L. (1993). Intrateam interdependence in task-performing groups. Journal of Applied Psychology, 78, 61-72.

Salas, E., Nichols, D. R. y Driskell, J. E. (2007). Testing three team training strategies in intact teams. Small Group Research, 38, 471-488.

Salas, E., Stagl, K. C. y Burke, C. S. (2004). 25 years of team effectiveness in organizations: Research themes and emerging needs. En C. L. Cooper y I. T Robertson 
(Eds.),International Review of Industrial and Organizational Psychology, Vol. 19 (pp. 47-91). Chichestter: John Wiley \& Sons.

Salas, E., Rosen, M. A., Burke, C. S. y Goodwin, G. F. (2009). The wisdom of collectives in organizations: An update of the teamwork competencies. En E. Salas, G.F. Goodwin y C.S. Burke (Eds.), Team Effectiveness in Complex Organizations. Cross-Disciplinary Perspectives and Approaches (pp. 39-79). Nueva York: Psychology Press.

Salas, E., Sims, D.E. y Burke, S. C. (2005). Is there a "Big Five" in teamwork? Small Group Research, 36, 555-599.

Salas, E., Stagl, K.C., Burke, S. C. y Goodwin, G. F. (2007). Fostering team effectiveness in organizations: Toward an integrative theoretical framework of team performance. En J. W. Shuart, W. Sapulding y J. Poland (Eds.), Modeling Complex Systems: Motivation, Cognition and Social Processes, Nebraska Symposium on Motivation (Vol. 51). Lincoln: University of Nebraska Press.

Salas, E. y Wildman, J. L. (2009). Ten critical research questions: The need for new and deeper explorations. En E. Salas, G. F. Goodwin y C.S. Burke (Eds.), Team Effectiveness in Complex Organizations. Cross-Disciplinary Perspectives and Approaches (pp. 525-546). Nueva York: Psychology Press.

Sánchez-Manzanares, M., Rico, R., Gibson, C. y Kearney, E. (En revisión). Shared mental models and performance in project teams: assessing the role of implicit coordination and task interdependence. Organization Science.

Schippers, M. C., Den Hartog, D. N. y Koopman, P. L. (2007). Reflexivity in teams: A measure and correlates. Applied Psychology: An International Review, 56, 189-211.

Scott, J. (2000). Social Network Analysis: A Handbook. NY:SAGE.

Shapiro, D. L., Furst, S. A., Spreitzer, G. M y von Glinow, M. A. (2002). Transnational teams in the electronic age: Are team identity \& high performance at risk? Journal of Organizational Behavior, 23, 455-67.

Shea, G. P. y Guzzo, R. A. (1987). Groups as human resources. En K. M. Rowland y G. R. Ferris (Eds.), Research in Personnel and Human Resources Management, Vol. 5 (pp. 323-356). Greenwich, CT: JAI.

Sims, D. E., Salas, E. y Burke, C. S. (2005). Promoting effective team performance through training. En S. A. Wheelan (Ed.), The Handbook of Group Research and Practice (pp.407-425). Thousand Oaks, CA: Sage.

Sivasubramaniam, N., Murry, W. D., Avolio, B. J. y Jung, D. I. (2002). A longitudinal model of the effects of team leadership and group potency on group performance. Group and Organization Management, 27, 66-96.

Smith-Jentsch, K. A., Mathieu, J. E. y Kraiger, K. (2005).Investigating linear and interactive effects of shared mental models on safety and efficiency in a field setting. Journal of Applied Psychology, 90, 523-535.

Snow, C. C., Lipnack, J. y Stamps, J. (2001). The virtual organization: promises and payoffs, large and small. En C. L. Cooper y D. M. Rousseau (Eds.) Trends in Organizational Behavior: The Virtual Organization (pp. 15-30). Chichester: Wiley.
Spreitzer, G. M., Cohen, S. G. y Ledford, G. E. Jr. (1999). Developing effective self-managing work teams in service organizations. Group y Organization Management, 24, 340-366.

Srivastava, A., Bartol, K. M. y Locke, E. A. (2006). Empowering leadership in management teams: Effects on knowledge sharing, efficacy, and performance. Academy of Management Journal, 49, 1239-1251.

Steiner, I. D. (1972). Group processes and productivity. New York: Academic Press.

Stewart, G. L. (2006). A meta-analytic review of relationships between team design features and team performance. Journal of Management, 31, 319-335.

Stewart, G. L. y Barrick, M. R. (2000). Team structure and performance: Assessing the mediating role of intrateam process and the moderating role of task type. Academy of Management Journal, 43, 135-148.

Sundstrom, E., McIntyre, M., Halfhill, T. y Richards, H. (2000). Work groups: from the Hawthorne studies to work teams of the 1990s and beyond. Group Dynamics: Theory, Research, and Practice, 4, 44-67.

Taggar, S., Hackett, R. y Saha, S. (1999). Leadership emergence in autonomous work teams: Antecedents and outcomes. Personnel Psychology, 52, 899-926.

Tasa, K., Taggar, S. y Seijts, G. H. (2007). The development of collective efficacy in teams: A multilevel and longitudinal perspective. Journal of Applied Psychology, 92, 17-27.

Tannenbaum, S. I., Beard, R. L. y Salas, E. (1992). Team building and its influence on team effectiveness: An examination of conceptual and empirical developments. Issues, Theory, and Research in Industrial/ Organizational Psychology, 82, 117-153.

Tesluk, P. E. y Mathieu, J. E. (1999). Overcoming roadblocks to effectiveness: Incorporating management of performance barriers into models of work group effectiveness. Journal of Applied Psychology, 84, 200-217.

Tesluk, P. E., Vance, R.J. y Mathieu, J. E. (1999). Examining employee involvement in the context of participative work environments. Group and Organization Management, 24, 271-299.

Tilin, F. y Sumerson, J.B. (2005).Team consultation. En S. A. Wheelan (Ed.), The Handbook of Group Research and Practice (pp.427-439). Thousand Oaks, CA: Sage.

Tjosvold, D., Hui, C., Ding, D. Z. y Hu, J. (2003). Conflict values and team relationships: Conflict's contribution to team effectiveness and citizenship in China. Journal of Organizational Behavior, 24, 69-88.

Tushman, M. y Nadler, D. (1996). Organizing for innovation. En K. Starkey (Ed.), How Organizations Learn (pp. 135-155). Londres: International Thomson Business Press.

Tyler, T. R. y Kramer, R. M. (1996). Whither trust? En R. M. Kramer y T.R. Tyler (Eds.) Trust in Organizations: Frontiers of Theory and Research (pp. 331-57). Thousand Oaks, CA: Sage.

van de Ven, A. H., Delbecq, L. A. y Koening, R. J. (1976). Determinants of coordination modes within organizations. American Sociological Review, 41, 322-338. 
van der Vegt, G.S . y Janssen, O.(2003). Joint impact of interdependence and group diversity on innovation. Journal of Management, 29, 729-751.

van der Vegt, G. S. y van de Vliert, E. (2005). Effects of perceived skill dissimilarity and task interdependence on helping in work teams. Journal of Management, 31, 7389.

van der Vegt, G. S., Emans, B. y van der Vliert, E. (2005). The congruence hypothesis of intrateam interdependence in organizations. En L. L. Neider y Ch. A. Schriesheim (Eds.) Understanding Teams. Greenwich, CN: Information Age Publishing.

vanKnippenberg, D. y Schippers, M. C. (2007). Work group diversity. Annual Review of Psychology, 58, 515-541.

Wageman, R. (2001). How leaders foster self-managing team effectiveness: Design choices versus hands-on coaching. Organization Science, 12, 559-577.

Wageman, R. B. y Baker, G.(1997). Incentives and cooperation: the joint effects of task and reward interdependence on group performance. Journal of Organizational Behavior, 18, 139-158.

Weingart, L. R. y Cronon, M. A. (2009). Teams research in the $21^{\text {st }}$ Century: A case for theory consolidation. En E. Salas, G. F. Goodwin y C.S. Burke (Eds.), Team Effectiveness in Complex Organizations. CrossDisciplinary Perspectives and Approaches (pp. 509-524). New York: Psychology Press.

West, G. W. (1996). Group learning in the workplace. En S. Imel (Ed.), Learning in Groups: Exploring Fundamental Principles, New Uses, and Emerging Opportunities (pp. 51-60). New Directions for Adult and Continuing Education, no. 71. San Francisco: Jossey Bass.

West, M. A. y Farr, J. L. (1990).Innovation at work. En M.A. West y J.L. Farr (Eds.), Innovation and creativity at work: Psychological and organizational strategies (pp. 3-13). Chichester, England: Wiley.

West, M. A. y Markiewicz, L. (2004).Building team-based working. A practical guide to organizational transformation.Oxford: BPS / Blackwell.

Wheelan S. A. (2009). Group size, group development, and group productivity. Small Group Research, 40, 247-262.

Wilke, H. A. M. y Meertens, R.W. (1994). Group performance. London: Routledge.
Williams, K. y O'Reilly, C. A. (1998). Demography and diversity in organizations: A review of 40 years of research. En B. M. Stawy L. L. Cummings (Eds.), Research in Organizational Behavior (pp. 77-140). Oxford, UK: Elsevier.

Wilson, J. M., Strauss, S. G. y McEvily, B. (2006). All in due time: the development of trust in computer-mediated and face-to-face teams. Organizational Behavior and Human Decision Processes, 99, 16-33.

Wittembaum, G. M., Stasser, G. y Merry, C. J. (1996). Tacit coordination in anticipation of small group task completion. Journal of Experimental Social Psychology, 32, 129-152.

Wojnar, L. y Uden, L. (2005). Group process and trust in group discussion. International Journal of Information and Communication Technology Education, 1, 55-68.

Wutchy, S., Jones, B. F. y Uzzi, B. (2007). The increasing dominance of teams in production of knowledge. Science, 316, 1036-1039.

Yang, J., Mossholder, K. W. y Peng, T. K. (2007). Procedural justice climate and group power distance: An examination of cross-level interaction effects. Journal of Applied Psychology, 92, 681-692.

Yoo, Y. y Kanawattanachai, P. (2001).Developments of transactive memory systems and collective mind in virtual teams. International Journal of Organizational Analysis, 9, 187-208.

Yu, C. P. y Chu, T. H. (2007). Exploring knowledge contribution from an OCB perspective. Information and Management, 44, 321-331.

Zaccaro, S. J., Rittman, A. L. y Marks, M. A. (2001). Team leadership. Leadership Quarterly, 12, 451-483.

Zalesny, M. D., Salas, E. y Prince, C. (1995). Conceptual and measurement issues in coordination: Implications for team behavior and performance. Research in Personnel and Human Resources Management, 13, 81-115.

Zellmer-Bruhn, M. y Gibson, C. (2006). Multinational organization context: Implications for team learning and performance. Academy of Management Journal, 49, 501518.

Zohar, D. (2000). A group-level model of safety climate: Testing the effect of group climate on microaccidents in manufacturing jobs. Journal of Applied Psychology, 85, 587-596.

Manuscrito Recibido: 21/12/2009

Revisión Recibida: 15/02/2010

Aceptado: 18/02/2010 